\title{
The cause of spatial structure in solar He I 1083 nm multiplet images ${ }^{\star}$
}

\author{
Jorrit Leenaarts $^{1}$, Thomas Golding ${ }^{2}$, Mats Carlsson ${ }^{2}$, Tine Libbrecht ${ }^{1}$, and Jayant Joshi ${ }^{1}$ \\ ${ }^{1}$ Institute for Solar Physics, Department of Astronomy, Stockholm University, AlbaNova University Centre, 10691 Stockholm, \\ Sweden \\ e-mail: jorrit.leenaarts@astro.su.se \\ 2 Institute of Theoretical Astrophysics, University of Oslo, PO Box 1029 Blindern, 0315 Oslo, Norway
}

Received 11 March 2016 / Accepted 2 August 2016

\begin{abstract}
Context. The He I $1083 \mathrm{~nm}$ is a powerful diagnostic for inferring properties of the upper solar chromosphere, in particular for the magnetic field. The basic formation of the line in one-dimensional models is well understood, but the influence of the complex threedimensional structure of the chromosphere and corona has however never been investigated. This structure must play an essential role because images taken in $\mathrm{He}$ I $1083 \mathrm{~nm}$ show structures with widths down to $100 \mathrm{~km}$.

Aims. We aim to understand the effect of the three-dimensional temperature and density structure in the solar atmosphere on the formation of the $\mathrm{He} \mathrm{I} 1083 \mathrm{~nm}$ line.

Methods. We solved the non-LTE radiative transfer problem assuming statistical equilibrium for a simple nine-level helium atom that nevertheless captures all essential physics. As a model atmosphere we used a snapshot from a 3D radiation-MHD simulation computed with the Bifrost code. Ionising radiation from the corona was self-consistently taken into account.

Results. The emergent intensity in the He I $1083 \mathrm{~nm}$ is set by the source function and the opacity in the upper chromosphere. The former is dominated by scattering of photospheric radiation and does not vary much with spatial location. The latter is determined by the photonionisation rate in the He I ground state continuum, as well as the electron density in the chromosphere. The spatial variation of the flux of ionising radiation is caused by the spatially-structured emissivity of the ionising photons from material at $T \approx 100 \mathrm{kK}$ in the transition region. The hotter coronal material produces more ionising photons, but the resulting radiation field is smooth and does not lead to small-scale variation of the UV flux. The corrugation of the transition region further increases the spatial variation of the amount of UV radiation in the chromosphere. Finally we find that variations in the chromospheric electron density also cause strong variation in He I $1083 \mathrm{~nm}$ opacity. We compare our findings to observations using SST, IRIS and SDO/AIA data.
\end{abstract}

Key words. Sun: atmosphere - Sun: chromosphere - radiative transfer

\section{Introduction}

The He I $1083 \mathrm{~nm}$ multiplet arises from the three radiative transitions between the $1 \mathrm{~s} 2 \mathrm{~s}^{3} \mathrm{~S}$ and $1 \mathrm{~s} 2 \mathrm{p}^{3} \mathrm{P}$ triplet terms of neutral helium. In the solar spectrum it appears as two absorption lines at $1082.91 \mathrm{~nm}$ and $1083.03 \mathrm{~nm}$ (in air). Because of the large energy difference between the lower level of the multiplet and the singlet ground state $(19.8 \mathrm{eV})$, the population of the lower level cannot be caused by electron collisions at typical chromospheric temperatures of $\sim 10 \mathrm{kK}$. This led Goldberg (1939) to propose that photoionisation from the He I ground state continuum $(\lambda<50.4 \mathrm{~nm})$ follow by spontaneous recombination (often called PR mechanism) is the driver of triplet population. Observational evidence from solar observations is the general correlation of X-ray emission and He I $1083 \mathrm{~nm}$ absorption (Kahler et al. 1983). Observations of weakly active dwarfs, giants and supergiant stars of spectral type F7 or later show a correlation between X-ray flux and He I $1083 \mathrm{~nm}$ equivalent width (Zarro \& Zirin 1986). The same relation has been established for active giants, but interestingly, not for active dwarfs (Sanz-Forcada \& Dupree 2008).

The observational evidence has been supported by many calculations of increasing sophistication (e.g. Zirin 1975; Livshits et al. 1976; Pozhalova 1988; Avrett et al. 1994;

\footnotetext{
* A movie associated to Fig. 4 is available at http://www . aanda.org
}

Andretta \& Jones 1997; Centeno et al. 2008), but all either in a plane-parallel slab geometry or using one-dimensional planeparallel atmospheres without an actual corona. Consequently the coronal radiation field in these calculations had to be prescribed, for which typically the data described in Tobiska (1991) has been used. All models reproduced the observed correlation between the solar extreme UV (EUV) flux and the He I $1083 \mathrm{~nm}$ equivalent width (EW, see Tobiska 1991). An interesting feature of the PR mechanism is that the $30.4 \mathrm{~nm}$ of He II line is the strongest line in the He I ground state continuum, so that the formation of the He I $1083 \mathrm{~nm}$ is influenced by the resonance lines of He II.

Livshits et al. (1976) demonstrated that the relative population density of the triplet system also depends on the electron density. By considering the dominant pathways that populate and depopulate the triplet system they found that the relative population of the triplet ground state $n\left(2 \mathrm{~s}^{3} \mathrm{~S}\right) / n_{\mathrm{He}}$ is approximately given by

$$
\frac{n\left(2 \mathrm{~s}{ }^{3} \mathrm{~S}\right)}{n_{\mathrm{He}}}=\frac{\alpha_{\mathrm{t}}}{\left(1+n_{\mathrm{e}} \alpha_{\mathrm{s}} / \phi_{\mathrm{s}}\right)\left(\phi_{\mathrm{t}} / n_{\mathrm{e}}+q_{\mathrm{ts}}\right)},
$$

with $\alpha_{\mathrm{t}}$ the photorecombination rate coefficient per electron into the triplet system, $n_{\mathrm{e}}$ the electron density, $\alpha_{\mathrm{s}}$ the photorecomination rate coefficient per electron into the singlet system, $\phi_{\mathrm{s}}$ and $\phi_{\mathrm{t}}$ the photoionization rate coefficients for the $1 \mathrm{~s}^{2}{ }^{1} \mathrm{~S}$ and $1 \mathrm{~s} 2 \mathrm{~s}^{3} \mathrm{~S}$ 
levels, and $q_{\mathrm{ts}}$ the electron collision rate coefficient per electron from the $1 \mathrm{~s} 2 \mathrm{~s}{ }^{3} \mathrm{~S}$ level into the $1 \mathrm{~s} 2 \mathrm{p}^{1} \mathrm{P}$ and $1 \mathrm{~s}^{2}{ }^{1} \mathrm{~S}$ singlet levels. The quantities $\alpha_{\mathrm{t}}$ and $\alpha_{\mathrm{s}}$ are approximately constant (exactly constant if stimulated recombination is ignored), $\phi_{\mathrm{s}}$ depends on the radiation field blueward of $50.4 \mathrm{~nm}, \alpha_{\mathrm{t}}$ depends on the radiation field blueward of $259 \mathrm{~nm}$, and $q_{\mathrm{ts}}$ depends on temperature.

The relative population is an increasing function of electron density for typical values of the upper chromospheric electron density $\left(10^{15}-10^{18} \mathrm{~m}^{-3}\right)$ and temperature $(10 \mathrm{kK})$ at fixed coronal UV illumination. We thus also expect variations in the He I $1083 \mathrm{~nm}$ strength caused by variations in the mass density and ionisation degree in the chromosphere.

An alternative mechanism for creating He I $1083 \mathrm{~nm}$ opacity is direct collisional excitation from the He I ground state to the lower level of the line. Owing to the large energy gap between the two levels this mechanism is inefficient in the chromosphere, but can act whenever the temperature is above $20000 \mathrm{~K}$, that is, in the lower transition region. (e.g. Milkey et al. 1973). At those temperatures one expects little He I based on statistical equilibrium calculations, but it might be present due to slow ionization and recombination during rapidly changing thermodynamic properties and the a drift of $\mathrm{He}$ I atoms into areas with higher electron temperature in the presence of strong temperature gradients and a long mean time between collisions. Several authors have proposed such mechanisms to explain the intensities of the resonance lines of helium, such as He I $53.7 \mathrm{~nm}$, He I $58.4 \mathrm{~nm}$, He II $30.4 \mathrm{~nm}$, and He II $25.6 \mathrm{~nm}$. (Milkey et al. 1973; Jordan 1975; Smith \& Jordan 2002; Smith 2003; Pietarila \& Judge 2004). Such effects also influence the formation of the He I $1083 \mathrm{~nm}$ in 1D radiation-hydrodynamics simulations (Golding et al. 2014).

The one-dimensional nature of the modelling efforts so far has meant that they are unable to explain the spatial structure of the He I $1083 \mathrm{~nm}$ equivalent width, besides the general conclusion that large EW corresponds to larger impinging EUV flux and/or larger chromospheric density.

High-resolution coronal images (e.g., Cirtain et al. 2013) show spatial structure down to $0^{\prime} ! 2$, indicating coronal temperature and/or density variations at least down to that scale, while the actual emitted spectrum below $50.4 \mathrm{~nm}$ strongly depends on temperature. This leads to a large variation in space of the emissivity of ionising radiation, and this should lead to a spatial variation of the ionising radiation impinging on the chromosphere. The transition region between the chromosphere and the corona is highly corrugated which will introduce additional differences in impinging flux as parts of the chromosphere can shield other parts from the coronal radiation.

Three-dimensional numerical simulations (e.g., Carlsson et al. 2016) as well as semi-empirical 1D models of the solar chromosphere (e.g., Fontenla et al. 2006) indicate that considerable density variations occur at the top of the chromosphere.

One thus expects strong spatial variation of the strength of the He I $1083 \mathrm{~nm}$. This is indeed the case: high-resolution observations show spatial structure down to a fraction of an arcsecond (e.g. Ji et al. 2012; Schad et al. 2013, 2015).

In this paper we investigate the causes of the spatial variation of the He I $1083 \mathrm{~nm}$ strength with help of numerical simulations of the solar atmosphere, 3D non-LTE radiative transfer calculations, and observations taken with the Swedish 1-m Solar Telescope (SST) and the Interface Region Imaging Spectrograph (IRIS). Our aim is to investigate in detail the origin of the variation in ionising UV radiation as well as the influence of the corrugation of the transition region and the chromospheric electron density variation on He I $1083 \mathrm{~nm}$ images.
In Sect. 2 we describe our numerical simulation and the subsequent radiative transfer modelling. In Sect. 3 we describe our observations. We describe the relation between coronal UV radiation, chromospheric electron density and He I $1083 \mathrm{~nm}$ absorption in Sect. 4. Section 5 contains a brief observational test of our theoretical finding that He I $1083 \mathrm{~nm}$ absorption should correlate with Si IV emission, and we finish with a discussion in Sect. 6.

\section{Numerical method}

Radiation-MHD simulation As a model atmosphere we use a snapshot from a three-dimensional radiation-MHD simulation computed with the Bifrost code (Gudiksen et al. 2011). This code solves the resistive-MHD equations together with heat conduction and non-LTE radiative losses on a Cartesian grid. The particular simulation that we use here is a $3 \mathrm{D}$ extension of the 2D simulations of Golding et al. (2016). It uses an equation-ofstate (EOS) that takes the non-equilibrium ionisation of hydrogen and helium into account. Non-equilibrium ionisation has a strong effect on the temperature and density structure in the chromosphere and transition region, and is thus required to obtain a realistic model of the upper chromosphere (Carlsson \& Stein 2002; Leenaarts et al. 2007; Golding et al. 2014, 2016).

The inclusion of the Lyman continuum and the Lyman alpha line in Bifrost is important because the hydrogen Lyman continuum is a source of ionising photons in the He I continuum. Treating these these transitions in detailed balance, as was done in earlier models (such as those described in Carlsson et al. 2016) leads to an erroneous amount of Lyman continuum photons in the subsequent radiative transfer step.

The simulation was run on a grid of $504 \times 504 \times 496$ points, with a horizontal domain size of $24 \times 24 \mathrm{Mm}^{2}$. In the vertical direction the domain spans from $2.4 \mathrm{Mm}$ below the average height of $\left\langle\tau_{500}\right\rangle=1$ to $12.4 \mathrm{Mm}$ above it. Besides the different EOS the run has an identical setup to the publicly available simulation described in Carlsson et al. (2016). In short, the simulation was run using an LTE EOS for $1750 \mathrm{~s}$ after an initial smooth bipolar magnetic field was introduced into a previously relaxed hydrodynamical simulation. This removed transients caused by the introduction of the magnetic field. Then the EOS was changed from LTE to non-equilibrium ionisation of hydrogen and helium, and the simulation was run for an additional $2000 \mathrm{~s}$. We took the snapshot $780 \mathrm{~s}$ after the non-equilibrium EOS was switched on. We refer the reader to Gudiksen et al. (2011), Golding et al. (2016), and Carlsson et al. (2016) for further details of the simulation.

Model atmosphere The Bifrost snapshot was reduced in size to a grid of $252 \times 252 \times 496$ by removing every second column in the two horizontal directions to reduce the computational cost of the radiative transfer computation.

Model atom We use the same helium model atom as Carlsson \& Stein (2002). By collapsing the sublevels in each term to a collective level this atom has only nine states. In this way the atom includes the $1 s^{2}, 1 s 2 s$, and $1 \mathrm{~s} 2 \mathrm{p}$ terms of the singlet system and the $1 \mathrm{~s} 2 \mathrm{~s}$ and $1 \mathrm{~s} 2 \mathrm{p}$ terms of the triplet system of He I, the 1 s, 2 s and 2 p terms of He II and a single state for He III. This model captures all pertinent processes despite its small size: it includes the bound-free transitions between the ground states that give rise to the ionisation edges at $22.8 \mathrm{~nm}$ and $50.4 \mathrm{~nm}$, the He II $30.4 \mathrm{~nm}$ line, and a representative He I $1083 \mathrm{~nm}$ line. The latter does not have the three components (from the upper levels $2{ }^{3} \mathrm{P}_{2}, 2{ }^{3} \mathrm{P}_{1}$, and $2{ }^{3} \mathrm{P}_{0}$ to the lower level $2{ }^{3} \mathrm{~S}_{1}$ ) as in reality, but 


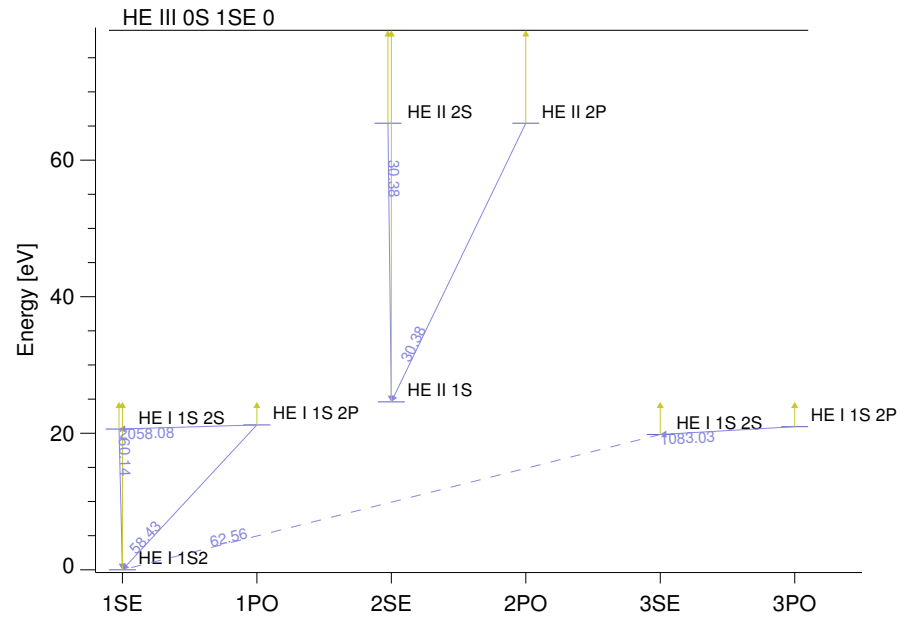

Fig. 1. Term diagram of our nine-level helium model atom. Solid blue lines indicate allowed radiative bound-bound transitions, dashed lines forbidden bound-bound transitions and yellowish lines radiative boundfree transitions.

instead consist of a single transition at $1083.33 \mathrm{~nm}$ in vacuum with an oscillator strength $f=0.539$. A term diagram of our model atom is shown in Fig. 1.

Radiative transfer The radiative transfer calculations are done with the Multi3d code (Leenaarts \& Carlsson 2009). It solves the problem of non-LTE radiative transfer in full 3D using the formalism of Rybicki \& Hummer (1991, 1992). The code is parallellized using MPI and employs domain decomposition in the three spatial dimensions as well as in frequency. The formal solution is computed on short characteristics using monotonic cubic Hermite polynomials (Auer 2003; Ibgui et al. 2013) to interpolate the source function and the extinction coefficient. Interpolation from grid points to cell interfaces is done using cubic convolution modified to avoid overshooting artefacts. As angle quadrature we use the A4 set from Carlson (1963), which contains 24 angles. We compute the background opacities, and background scattering probabilities in the photosphere and chromosphere for all elements except hydrogen and helium in LTE using the Uppsala opacity package (Gustafsson 1973). The hydrogen opacities and scattering probabilities are based on the non-equilibrium hydrogen populations from the Bifrost simulation (see Sect. 2) instead. We note that the background opacities include a scattering term assuming coherent scattering instead of complete redistribution. This assumption influences the Lyman-continuum intensities, which in turn has an effect on the photoionisation in the He I ground state continuum. Ideally one would like to run both hydrogen and helium simultaneously in full non-LTE, but this is currently not possible with Multi3d.

Applicability of statistical equilibrium for helium In Golding et al. (2014, 2016) we showed that the ionisation of helium is out of statistical equilibrium. Comparisons of one-dimensional calculations assuming $\mathrm{SE}$ and $\mathrm{NE}$ using the RADYN code (e.g., Carlsson \& Stein 1992) indicate that significant differences in the He I $1083 \mathrm{~nm}$ profiles can occur between SE and NE calculations (see Fig. 9 of Golding et al. 2014). We therefore expect that the current SE calculations will be substantially different from a more physically correct NE calculation. The latter is however currently beyond our means. We focus here on the spatial structure of He I $1083 \mathrm{~nm}$ images caused by the spatial variation in the irradiation from the corona and variations in the chromospheric electron density, which will be qualitatively reproduced by our SE approach. We do not attempt a detailed analysis of the line profiles because they will be quantitatively affected by NE effects.

Coronal radiation We include radiation emitted in the corona by adding a coronal emissivity:

$\psi_{v}=\Lambda_{v}(T) n_{\mathrm{e}} n_{\mathrm{H}}$,

where $\psi_{v}$ is the emissivity in units of power per solid angle per frequency per volume, $n_{\mathrm{e}}$ and $n_{\mathrm{H}}$ are the electron density and hydrogen density and $\Lambda_{v}(T)$ is the emissivity per hydrogen atom per electron. The quantity $\Lambda_{v}(T)$ was computed using the CHIANTI package version 7.1 (Dere et al. 1997; Landi et al. 2013). For a grid of 121 temperatures, logarithmically spaced between $1 \mathrm{kK}$ and $10 \mathrm{MK}$, we computed the radiative losses assuming coronal equilibrium ionisation balance for all lines in the CHIANTI database using the abundance given in the file sun_coronal.abund and the ion fractions given in the file chianti.ioneq. We removed all lines from hydrogen and helium because they are accounted for already in Multi3d: helium as the active non-LTE atom and hydrogen as a background opacity source. The resulting emissivity was binned into $0.1 \mathrm{~nm}$ wide bins such that the frequency integral yields the total radiative losses at that temperature. This table was remapped to the coarser frequency grid used in Multi3d while conserving the frequency integral.

The ionisation state in the time-varying corona is not in equilibrium (e.g., Joselyn et al. 1979; Hansteen 1993; Bradshaw \& Raymond 2013), and this will have an effect on the coronal emissivities. While it is possible to run Bifrost with non-equilibrium coronal ionisation (Olluri et al. 2013b), it is computationally prohibitively expensive to include all elements and atomic levels present in the CHIANTI line list. We therefore opted for the simpler and faster coronal equilibrium approximation.

\section{Observations}

The observations were taken on 2015-07-31 from 08:46:36 UT to 08:50:18 UT with the TRIPPEL spectrograph (Kiselman et al. 2011) at the Swedish 1-m Solar Telescope (SST, Scharmer et al. 2003) on La Palma. We obtained raster scans of active region NOAA 12393 at a viewing angle $\mu=0.76$, recorded in reasonable but not excellent seeing conditions. The raster scan contains 300 slit positions with a step size of $0{ }^{\prime \prime} 1$ and a slit width of $00^{\prime \prime} 11$. The field-of-view is $30^{\prime \prime} \times 34^{\prime \prime}$. 2 . It took $222 \mathrm{~s}$ to record the full scan.

The SST was used for the first time to observe in the infrared, employing an OWL-camera (OWL SW1.7 CL-640). The recorded spectral region ranges from $1081.84 \mathrm{~nm}$ to $1083.49 \mathrm{~nm}$ featuring the He I $1083 \mathrm{~nm}$ and Si I $1082.7 \mathrm{~nm}$ spectral lines. The estimated spectral resolution of TRIPPEL is $\lambda / \delta \lambda \approx 150000$ as determined by comparing with a high resolution reference spectrum (Kiselman et al. 2011).

The sensor of the OWL-camera has a size of $640 \times 512$ pixels with a spectral dispersion of $2.6 \mathrm{pm} / \mathrm{pixel}$ and a spatial scale of 0 '.067 pixel $^{-1}$. The spectra were rebinned in the spatial direction to a scale of 0.1 arcsec/pixel to create square pixels in the raster scan. We took three acquisitions in each slit position with an exposure time of $100 \mathrm{~ms}$ each, with a $0.74 \mathrm{~s}$ cadence per slit 
position. For each slit position we used the acquisition that had maximum continuum contrast.

We reduced the spectra using the TRIPPEL pipeline (Pereira et al. 2009; Kiselman et al. 2011). The reduction steps consist of flat field and dark frame corrections, geometrical corrections for smile and keystone and a wavelength calibration. The spectra were corrected for stray-light and hot pixels were removed from the spectra.

The SST observations were accompanied by observations with the Interface Region Imaging Spectrograph (IRIS, De Pontieu et al. 2014). The IRIS continuum channel at $283.2 \mathrm{~nm}$ was used for alignment with the SST raster scan continuum image: first, the IRIS slit-jaw images were interpolated to the scale of the SST raster scan, rotated and aligned with the slit jaw image. Second, to correct for higher-order shifts due to seeing, we applied a destretching routine from the CRISPRED pipeline (de la Cruz Rodríguez et al. 2015) to the SST raster scan with the seeing-free IRIS $283.2 \mathrm{~nm}$ continuum image taken half-way during the SST scan as an anchor. As a result of this correction, we are confident that the alignment has accuracy of $\sim 0^{\prime \prime} .2$ which is below the IRIS resolution of $\sim 0^{\prime \prime} .35$.

We also make use of co-spatial and co-temporal data taken with the Atmospheric Imaging Assembly (AIA, Lemen et al. 2012) on board the Solar Dynamics Observatory (SDO, Pesnell et al. 2012). These data were co-aligned with the IRIS and SST data to within an accuracy of one AIA pixel.

In this paper we compare the SST raster scan with IRIS Si IV $140 \mathrm{~nm}$ slit-jaw images (from now on Si IV SJI) and the AIA data. Therefore, we created an artificial raster scan with the same slit positions and cadence as the SST raster scan from the Si IV SJI images. Each column in this artificial raster scan equals the column in the Si IV SJI which is the nearest neighbour in time to the SST acquisition time of the column. Due to the big difference in cadence $(0.74 \mathrm{~s}$ per slit position in the SST and $17 \mathrm{~s}$ cadence for the Si IV SJI), only 14 slit-jaw images are used to compose this artificial raster scan. Artificial rasters of the AIA data (12 s cadence) where created in the same way as was done for the IRIS data.

\section{Results}

\subsection{Coronal sources of ionising radiation in the He I ground state continuum}

Our 3D Bifrost model includes the lower corona with variations in temperature and density, so it should mimic the observed behaviour. We investigate systematically how the EUV radiation is produced.

As a first check we compared the average UV flux in our model to the values in the "low solar activity model" of Tobiska (1991). Newer models are available (see Tobiska 2004), but the 1991 model is sufficiently accurate for our purposes. We integrated the average vertically emerging intensity in our model from $14.3 \mathrm{~nm}$ to $50.4 \mathrm{~nm}$, the range over which we include absorption and emission in the He I ground state continuum, and found a value of $48 \mathrm{~W} \mathrm{~m}^{-2}$ ster $^{-1}$. This is $\sim 60 \%$ higher than the corresponding value computed from Tobiska (1991). We thus conclude that our model produces a UV flux in the He I ground state continuum comparable to observations.

We note that we do not include absorption in the He I continuum below $14.3 \mathrm{~nm}$, for which Tobiska gives $3.7 \mathrm{~W} \mathrm{~m}^{-2} \mathrm{ster}^{-1}$, so the error is small. Also note that the Tobiska results are consistent with newer observations with AIA/EVE (Woods et al. 2012).
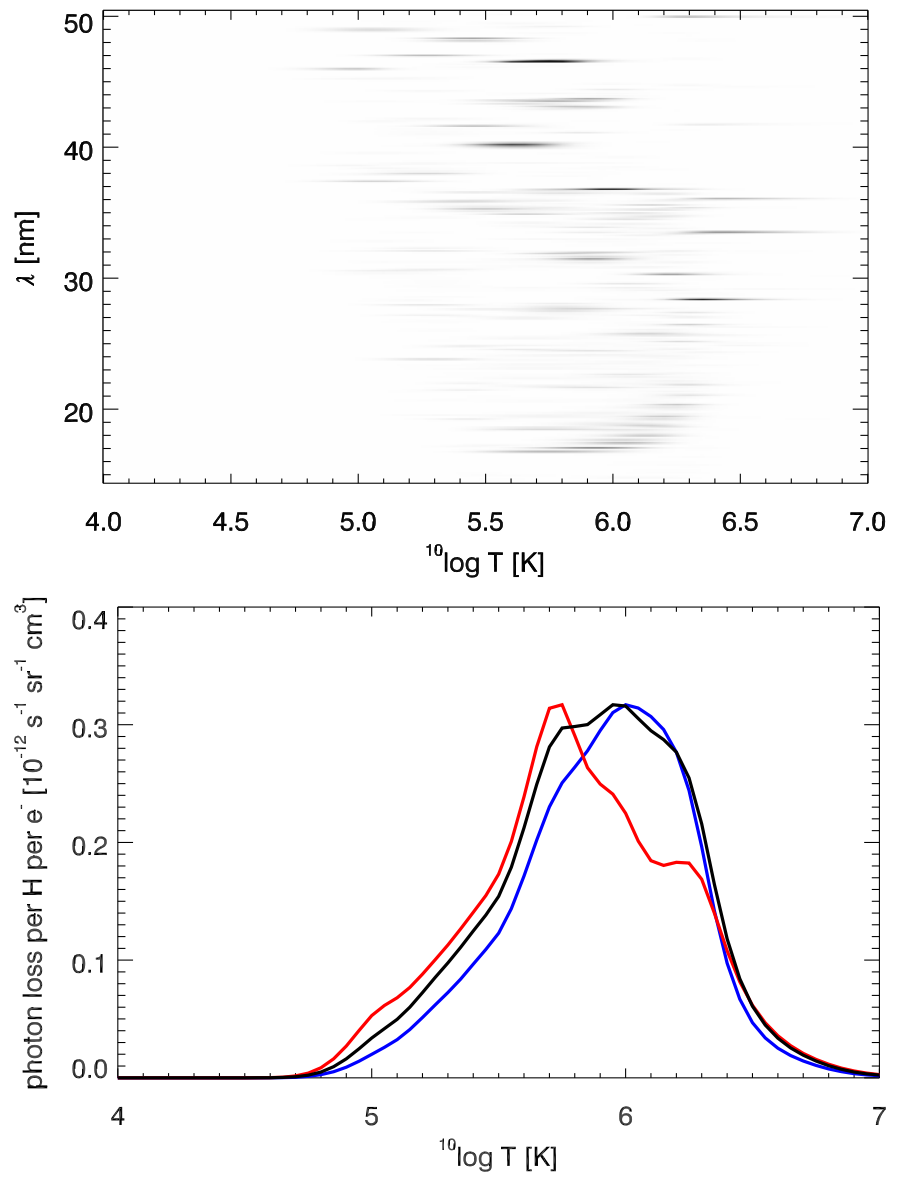

Fig. 2. Top: coronal radiation losses per hydrogen atom per electron $\left(\Lambda_{v}(T)\right.$ in Eq. (2)) as function of temperature and wavelength. The scale is inverted, so black means large losses. Bottom: $\Lambda_{\mathrm{E}}$, the frequencyintegrated energy losses per hydrogen atom per electron (blue, arbitrary units); $\Lambda_{\mathrm{ph}}$, the frequency-integrated photon losses per hydrogen atom per electron (black, scale on the left); $\Lambda_{\text {csw }}$, the frequency-integrated, He I ground-state continuum cross section weighted, photon losses per hydrogen atom per electron (red, arbitrary units).

In Fig. 2 we show $\Lambda_{v}(T)$ in Eq. (2) for $\lambda<51 \mathrm{~nm}$. The strongest emission feature is a Ne VII resonance line at $46.522 \mathrm{~nm}$ at $\log _{10} T=5.7$. The blue curve in the bottom panel shows $\Lambda_{E}=\int_{v_{\min }}^{v_{\max }} \Lambda_{v} \mathrm{~d} v$, the losses integrated over the frequency range in which we include He I ground state continuum opacity in our model atom. This distribution peaks at $\log _{10} T=6$.

In black we show the frequency-integrated photon losses, to take into account that each emitted photon can only ionise one He I atom (we omit the integration bounds for brevity):

$\Lambda_{\mathrm{ph}}=\int \frac{\Lambda_{v}(T)}{h v} \mathrm{~d} v$.

The photon-energy weighting increases the relative contribution of lower-temperature gas to the He I photoionisation because of the weighting with the inverse frequency. Finally we computed the coronal photon losses as the He I ground state continuum experiences it, by also weighting with the photoionisiaton cross section:

$\Lambda_{\mathrm{csw}}=\int \sigma_{v} \frac{\Lambda_{v}(T)}{h v} \mathrm{~d} v$,

shown in red in the bottom panel of Fig. 2. The peak of the distribution then shifts to ${ }^{10} \log T=5.7 \mathrm{~K}$, owing to the Ne VII line. 

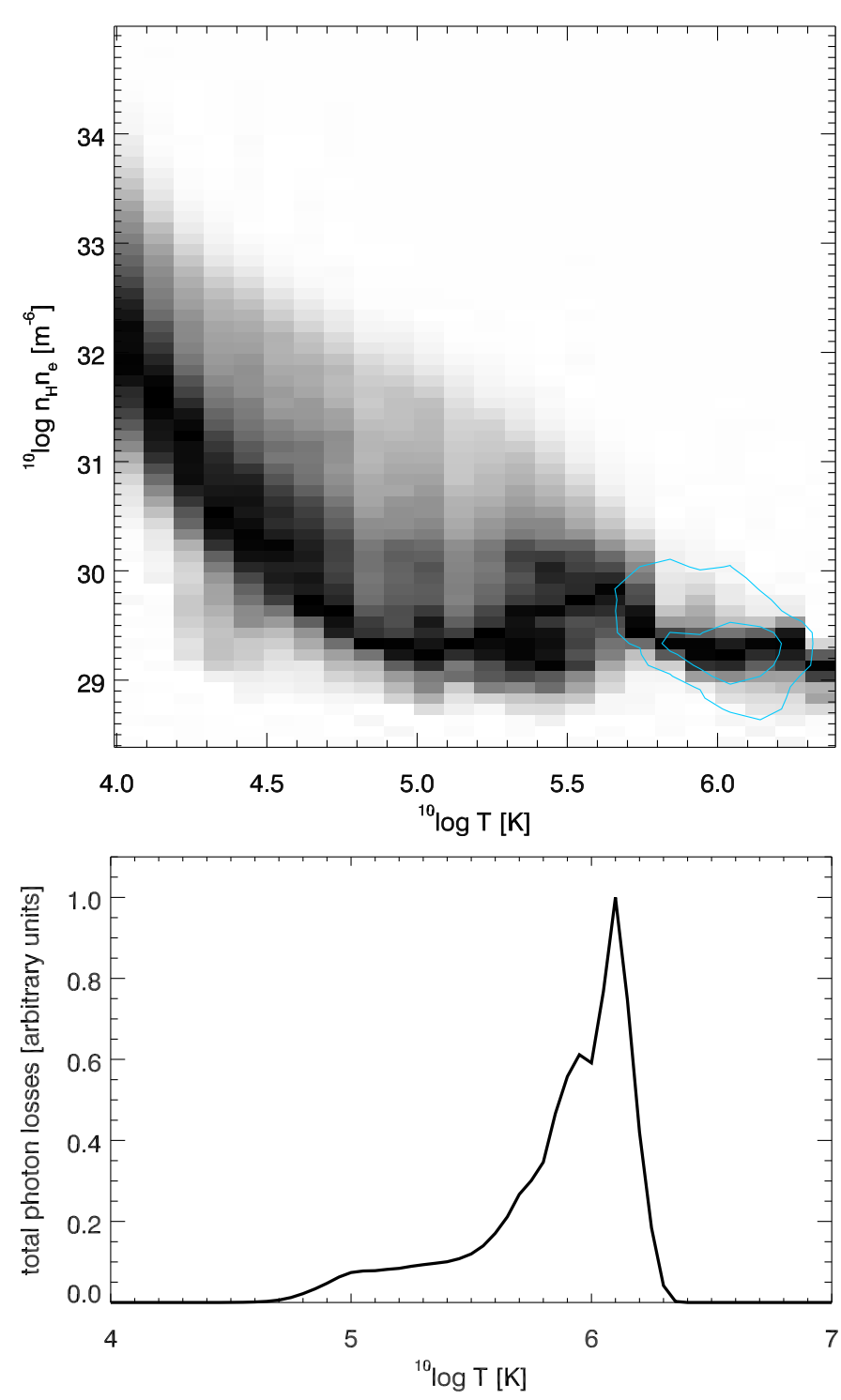

Fig. 3. Top: scaled joint probability density functions (JPDF) of $n_{\mathrm{H}} n_{\mathrm{e}}$ versus $T$ in the chromosphere and corona of our simulation snapshot. The inner blue contour includes $50 \%$ of all pixels, the outer contour $75 \%$. Each column in the panels is scaled to maximum contrast to increase visibility. Bottom: volume-integrated, frequency integrated and cross-section weighthed photon losses as function of temperature in our simulation snapshot (i.e., $\int_{V} L_{\mathrm{csw}} \mathrm{d} V$ in Eq. (5)).

In summary, the He I ground state continuum is sensitive to radiation emitted at a wide range of temperatures, with peak sensitivity at $500 \mathrm{kK}$ plasma.

We now proceed to investigate the effect of the density variations in the transition region and corona of the atmosphere model. Figure 3 shows the distribution of $n_{\mathrm{H}} n_{\mathrm{e}}$ versus the temperature in the chromosphere and corona of our simulation snapshot. The majority of the volume is filled with gas at coronal temperatures, but this gas has a relatively low density. The colder material at transition-region temperatures occupies a much smaller volume, but has a much higher density. Combining all ingredients we finally arrive at the bottom panel of Fig. 3 , which shows

$$
\int_{V} \Lambda_{\mathrm{csw}} n_{\mathrm{H}} n_{\mathrm{e}} \mathrm{d} V=\int_{V} L_{\mathrm{csw}} \mathrm{d} V
$$

the cross-section weighted total production of ionising photons in our simulation volume $V$ as function of temperature. The majority of the photons are produced by the tenuous coronal gas but there is a significant tail toward transition region temperatures.

In Fig. 4 we show the effect of the spatial variation of $L_{\mathrm{csw}}$. Panels a-c show $L_{\mathrm{csw}}$ integrated along the $x, y$, and $z$-axis, respectively. The distribution along $2 \mathrm{D}$ cross cuts are given in the accompanying animation. The emission (red colours in the animation) is highly structured, and peaks in a thin sheath just above the chromosphere.

The resulting cross-section weighted radiation field $J_{\text {csw }}$ impinging on the chromosphere is shown in panel d of Fig. 4. It shows a good correlation with the locations of strong emission on large scales, but not on small scales. This is because the radiation field at location $\boldsymbol{r}$ is in essence the integral of the emissivity weighted with the inverse distance squared:

$J_{\mathrm{csw}}(\boldsymbol{r}) \approx \int_{0}^{\infty} \int_{V} \frac{4 \pi}{h v} \sigma_{v} \frac{\psi_{v}\left(\boldsymbol{r}^{\prime}\right)}{\left|\boldsymbol{r}-\boldsymbol{r}^{\prime}\right|^{2}} \mathrm{e}^{-\tau\left(\boldsymbol{r}, \boldsymbol{r}^{\prime}\right)} \mathrm{d} \boldsymbol{r}^{\prime} \mathrm{d} v$,

with $\tau\left(\boldsymbol{r}, \boldsymbol{r}^{\prime}\right)$ the optical thickness between points $\boldsymbol{r}$ and $\boldsymbol{r}^{\prime}$, and the integral over $\boldsymbol{r}^{\prime}$ is taken over all space. The emissivity is mainly due to the corona where the extinction is negligible, and the extinction is essentially chromospheric. Therefore $J_{\text {csw }}$ is sensitive to both strong, closely located sources as well as larger regions at larger distances. The column-integrated lower level population of the He I $1083 \mathrm{~nm}$ shows a similar large scale correlation (panel e, the actual level population per volume is shown in blue in the animation). In panel $\mathrm{f}$ we show the resulting image at the nominal line centre wavelength, which shows fine structuring up to a scale of a fraction of a $\mathrm{Mm}$.

We note that the term $\mathrm{e}^{-\tau\left(\boldsymbol{r}, \boldsymbol{r}^{\prime}\right)}$ contains significant complexity. It encodes the different opacity at different wavelengths, not only for helium but also for the main other absorber, which is the hydrogen Lyman continuum. Assuming all hydrogen and helium neutral, then the He I ground state continuum makes up $40 \%$ of the total chromospheric opacity at $50 \mathrm{~nm}$ and $70 \%$ at $15 \mathrm{~nm}$. Our analysis thus somewhat overestimates the effect of longwavelength photons, because $\Lambda_{\mathrm{csw}}$ does not take this wavelength dependence into account.

Figure 4 and the animation show that the high emissivity points are located close to the chromosphere These will thus contribute most to the high $J_{\text {csw }}$ in the nearby chromosphere. In Fig. 5 we show the temperature distribution of the high- $L$ points. It peaks at $100 \mathrm{kK}$, indicating that the emission is coming from the transition region. Inspection of the cross-section-weighted emissivities computed with CHIANTI at $T=100 \mathrm{kK}$ and $n_{\mathrm{e}}=10^{16} \mathrm{~m}^{-3}$ show that only 37 lines of C III, C IV, N III, Ne II, Ne III, Ne IV, and C III produce $88 \%$ of the ionising photons.

In summary, we have shown that in our model the main source of the small-scale variation of ionizing radiation in the He I ground state continuum is a thin shell of relatively dense material at $100 \mathrm{kK}$ in the transition region. Whenever a pocket of chromospheric material is sufficiently illuminated by this material it develops appreciable opacity in the He I $1083 \mathrm{~nm}$. This mainly occurs in material that is almost completely surrounded by TR material, that is, slender extensions of the chromosphere that protrude into the corona.

\subsection{Other sources of ionizing UV radiation}

Besides radiation from bound-bound transitions in the TR and corona, there are other sources of ionising radiation. The most important contributions are the He II ground state continuum, 

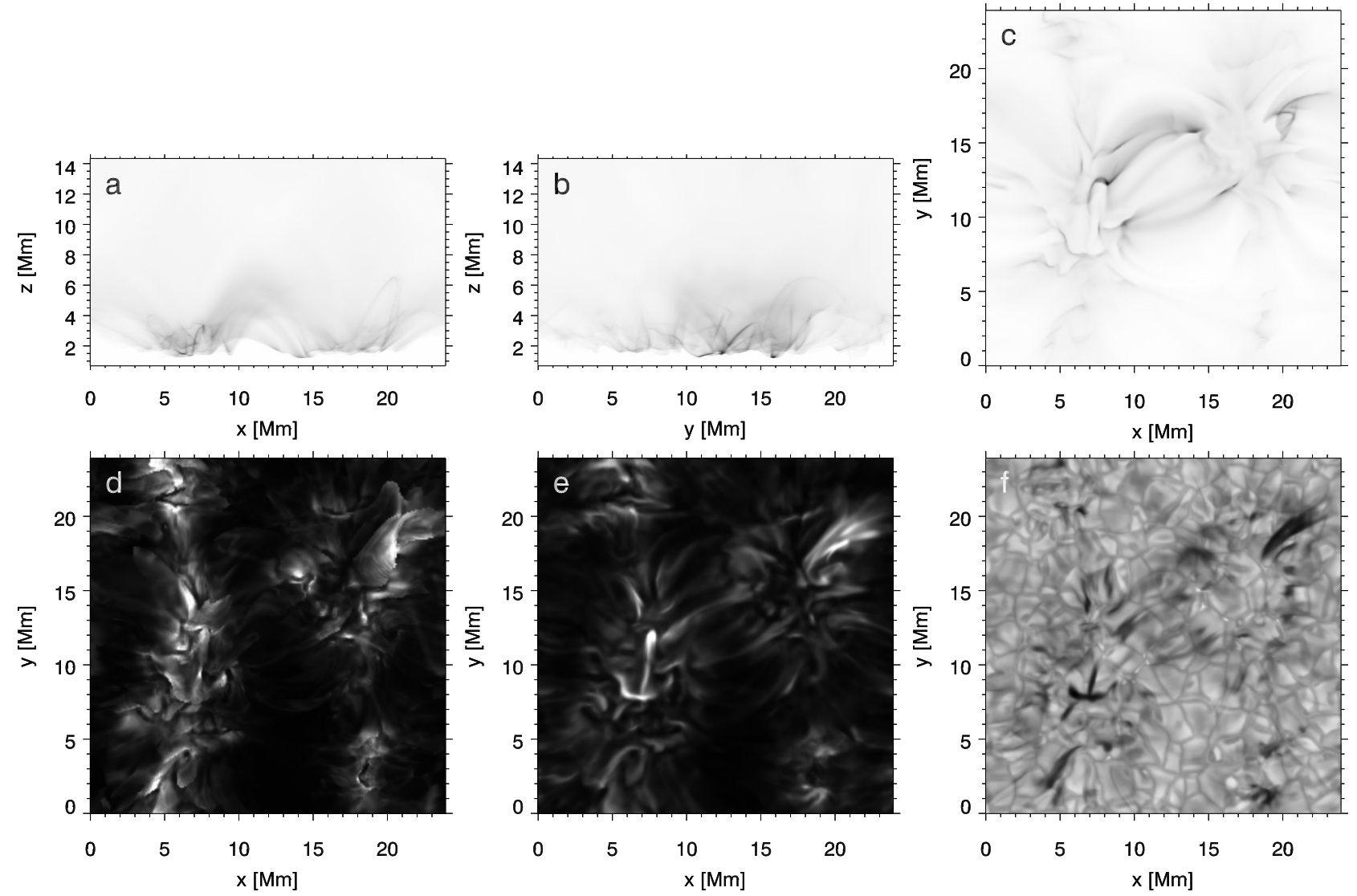

Fig. 4. Spatial relation between coronal emissivity and opacity in He I $1083 \mathrm{~nm}$. Top row: frequency integrated and cross-section weighted photon losses $L_{\mathrm{csw}}$, integrated along the $y$-axis a); the $x$-axis b) and the $z$-axis c); on an inverted brightness scale. Panel d): the resulting angle-averaged radiation field at the largest height in each column where $T=15 \mathrm{kK}$, showing the spatial variation of the ionising radiation impinging on the chromosphere. Panel e): total depth-integrated column density of the lower level of the He I $1083 \mathrm{~nm}$. Panel f): vertically emergent intensity at the nominal line core of the He I $1083 \mathrm{~nm}$. This figure has an accompanying movie available online. The movie shows 2D cross-cuts through the simulation domain, with $\Lambda_{\mathrm{csw}}$ in red and the He I $1083 \mathrm{~nm}$ lower level population in blue.

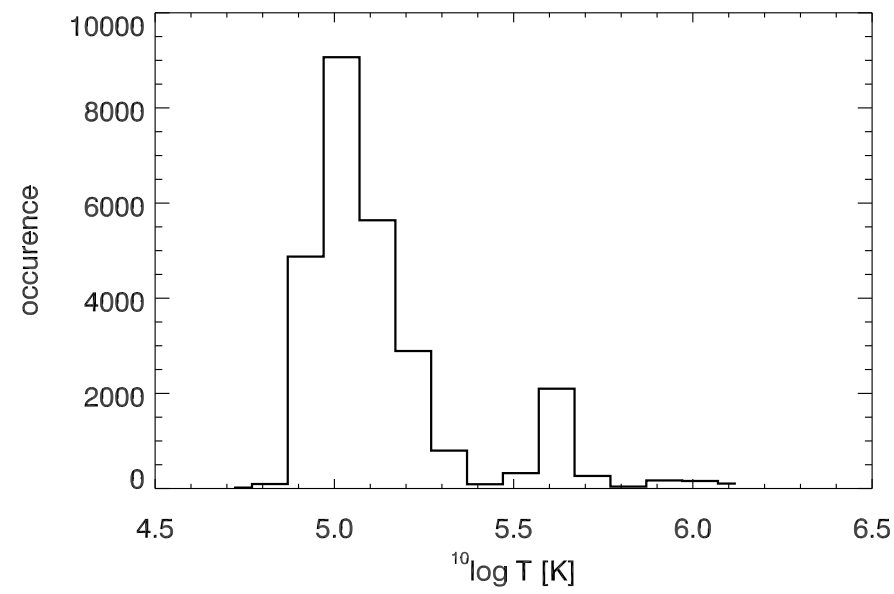

Fig. 5. Histogram of the temperature of the gas for grid points in the model with $\left.L_{\mathrm{csw}}>0.01 \max \left(L_{\mathrm{csw}}\right)\right)$.

the He II $30.4 \mathrm{~nm}$ line and the hydrogen Lyman continuum. The first two are included self-consistently in statistical-equilibrium non-LTE because they are included in the model atom. The latter is included as a background element in the following way: the hydrogen level populations are taken from the Bifrost model, where they were computed including non-equilibrium ionisation effects (Golding et al. 2016). The Lyman continuum is treated as a background process in our approximation, which means we assume coherent scattering and a photon destruction coefficient computed using the Van Regemorter approximation. We solved the 3D non-LTE radiative transfer problem both with and without the coronal emissivities to investigate the importance of the hydrogen Lyman and He II continua on the He I $1083 \mathrm{~nm}$ opacity.

Figure 6 compares both computations. Ignoring the coronal emissivity leads to much lower total chromospheric column density, and thus optical thickness, for the He I $1083 \mathrm{~nm}$, on average a factor 6.3 less (panels a and b). The resulting optical thickness is too low to leave a strong visible imprint in the intensity images at the line core. Only at a few locations is the photoionisation of $\mathrm{He}$ I by He II and H I radiation strong enough to be clearly visible. The best example is the upside-down T-shaped dark feature at $(x, y)=(7,8) \mathrm{Mm}$.

The frequency-integrated spatially-averaged intensity of the He II $30.4 \mathrm{~nm}$ line in our model is $13 \mathrm{~W} \mathrm{~m}^{-2} \mathrm{ster}^{-1}$, which is about $27 \%$ of the total intensity between 14.3 and $50.4 \mathrm{~nm}$. This is somewhat higher than the observed values of MacPherson \& Jordan (1999), who measured $5 \mathrm{~W} \mathrm{~m}^{-2} \mathrm{ster}^{-1}$ for internetwork and $10 \mathrm{~W} \mathrm{~m}^{-2}$ ster $^{-1}$ for a strong network boundary. So while our computation of the He II $30.4 \mathrm{~nm}$ line might be deficient in the sense that we ignore non-equilibrium effects, it nevertheless produces roughly the right intensity. We thus conclude that the line is an important, but not dominant, source of ionizing photons in the He I ground state continuum. 

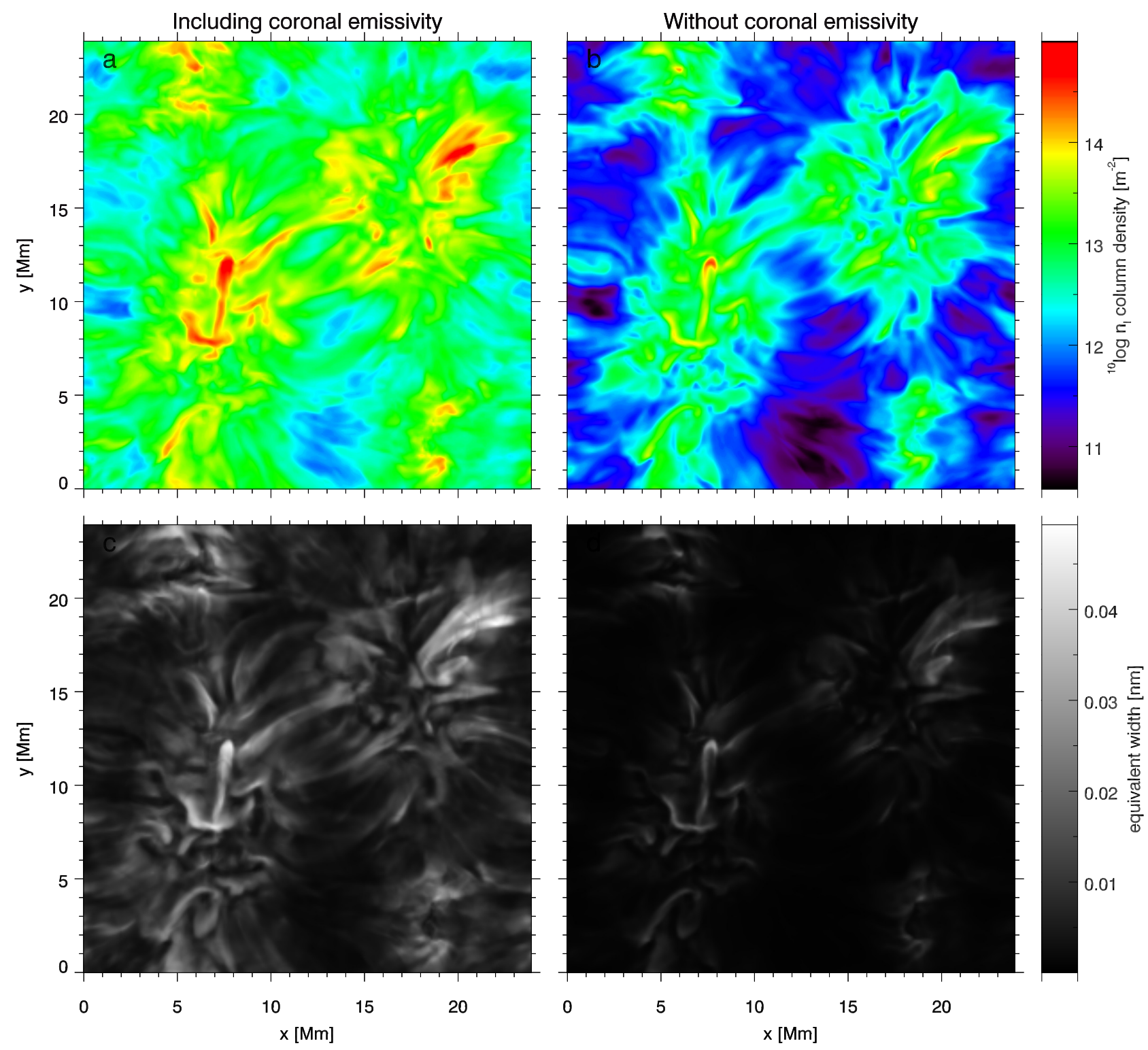

Fig. 6. Demonstration of the importance of radiation from the corona and transition region for the photoionisation in the He I ground state continuum. Panels a) and b) compare the column integrated lower-level population of the He I $1083 \mathrm{~nm}$ line with a) and without b) the inclusion of coronal and TR emission. Panels c) (with emission) and d) (without emission) compare the resulting equivalent widhts of the He I $1083 \mathrm{~nm}$ line.

\subsection{Importance of direct collisional excitation}

We computed the $N_{\text {hot }}$, total number of He I atoms in the lower level of the He I $1083 \mathrm{~nm}$ line in our model chomosphere, transition region and corona, for those grid points with a temperature above $20 \mathrm{kK}$, and computed $N_{\text {cold }}$, the same quantity but now for those points where $T<20 \mathrm{kK}$. We find that $N_{\text {hot }} / N_{\text {cold }}=0.005$, and thus conclude that direct collisional excitation is an insignificant source of He I $1083 \mathrm{~nm}$ opacity in our statistical equilibrium computation.

\section{4. $3 D$ effects on the UV illumination}

The coronal UV radiation spreads out in 3D. We test the importance of the horizontal spreading by comparing a computation using the full 3D radiation field (3D) and a computation where each column in the model atmosphere is treated as an independent plane-parallel atmosphere (1D). In Fig. 7 we show the differences between the two computations.

Panels $\mathrm{a}$ and $\mathrm{b}$ compare the UV radiation field in a vertical slice through the model. Panel b show vertical striping in $J$, typical for $1 \mathrm{D}$ calculations, whereas the $3 \mathrm{D}$ computations has a more even distribution. Notice however that the 3D computation also shows artefacts: the diagonal striping is caused by the finite number of ray angles that we use (24 angles). A small volume with high emissivity will thus only illuminate the surrounding space along the ray directions. Because the coronal emissivity is highly structured this shows up strongly. The middle row compares the resulting He I $1083 \mathrm{~nm}$ lower level populations along the same vertical slice. In 3D the population is much higher than in $1 \mathrm{D}$. This is mainly due to the effective self-shielding in 1D geometry, because radiation can only come from one direction. In 3D extensions of chromospheric material into the corona can be illuminated from all sides. A clear example can be seen at $(x, z)=(7.5,2.0) \mathrm{Mm}$, where a finger of chromospheric material 
$3 \mathrm{D}$
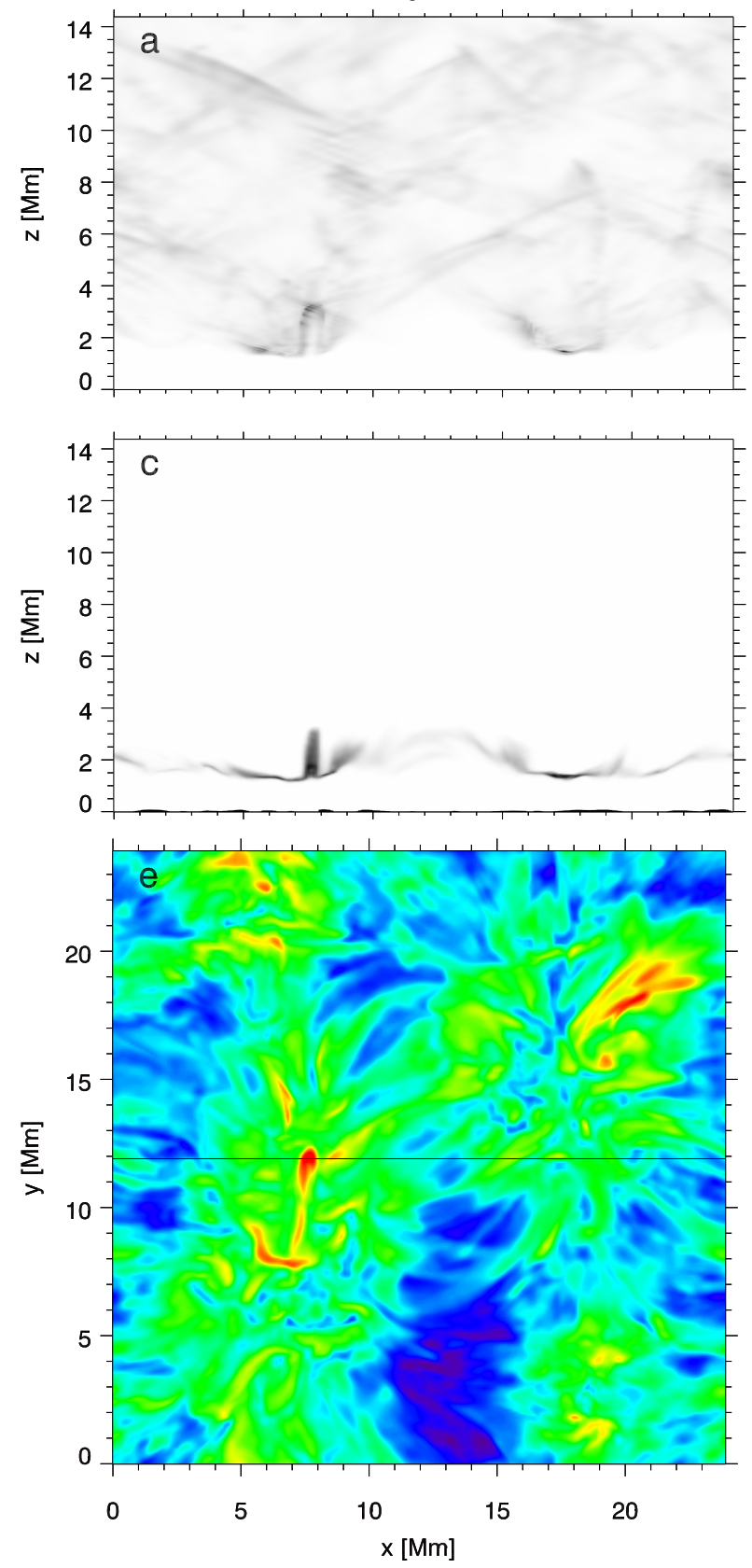

$1 \mathrm{D}$
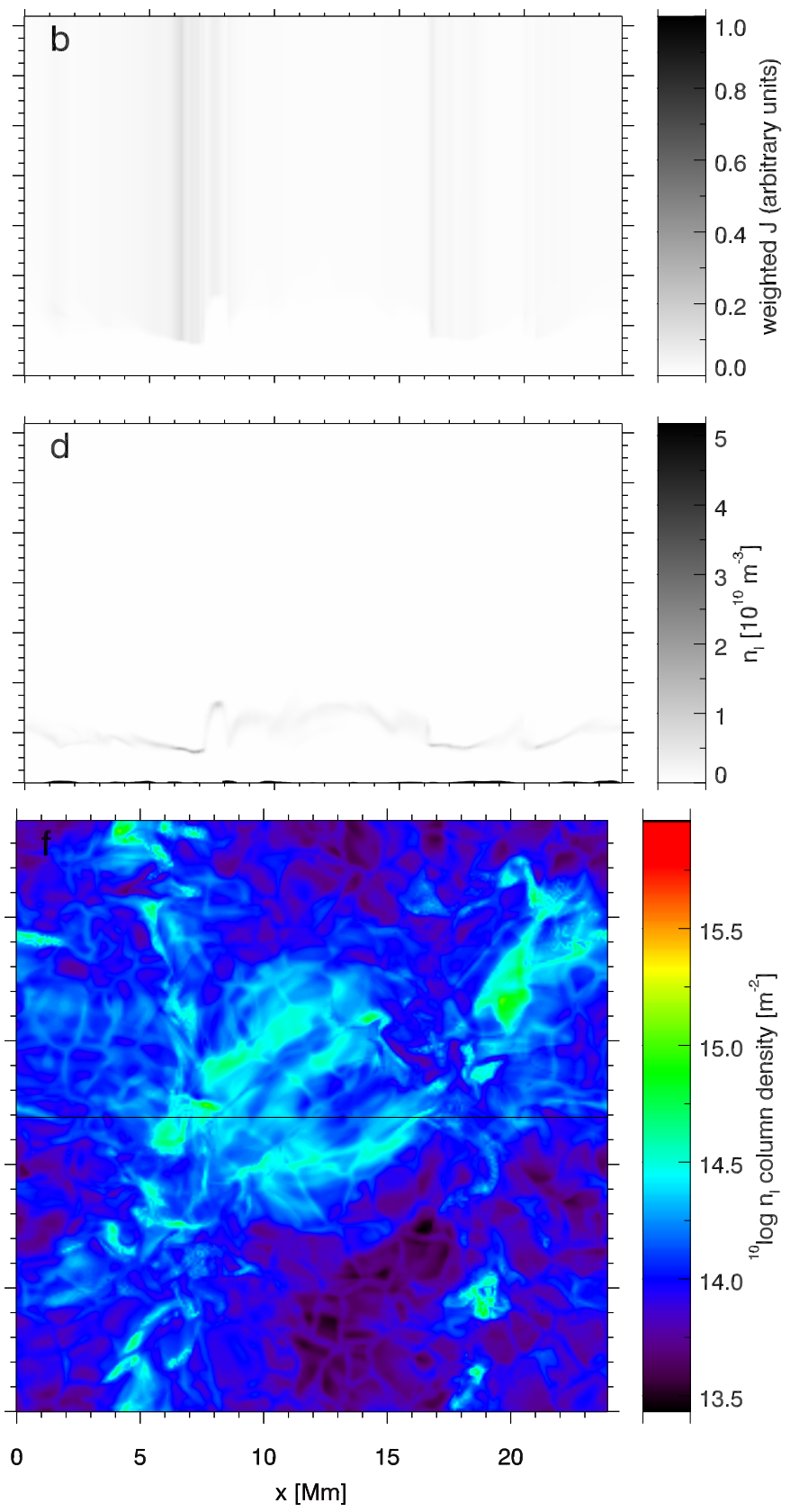

Fig. 7. Comparison of the He I $1083 \mathrm{~nm}$ lower-level population assuming 3D radiative transfer (left-hand column) and 1D radiative transfer (righthand column). Top row: cross-section weighted angle-averaged radiation field in a vertical slice at $y=11.9 \mathrm{Mm}$. Middle row: resulting lower level population of the He I $1083 \mathrm{~nm}$ line in a vertical slice at $y=11.9 \mathrm{Mm}$. Bottom row: column integrated lower-level population of the He I $1083 \mathrm{~nm}$ line, with the slice used in panels a)-d) indicated with a black line.

is surrounded by high-emissivity TR/coronal material. Similar effects were already reported by Carlsson \& Leenaarts (2012), who investigated 3D effects on the heating of the chromosphere by UV radiation produced in the corona.

Finally, in panels e and $f$ we compare the spatial distribution of the total column density of the lower-level population of the He I $1083 \mathrm{~nm}$ line. In 3D it is on average 4.2 times higher than in $1 \mathrm{D}$, and its maximum is 6.8 times higher.

\subsection{D effects on the source function}

At the height in the chromosphere where the He I $1083 \mathrm{~nm}$ line has appreciable opacity, the mass density is so low that the damping wings of the Voigt profile are negligible, and the extinction coefficient is essentially Gaussian. The photon destruction probability $\epsilon$ in the He I $1083 \mathrm{~nm}$ is of the order $10^{-4}-10^{-2}$. The line is thus scattering dominated $\left(S_{v} \simeq \bar{J}_{v 0}\right)$ and the thermalisation depth is located at a line core optical depth $\tau \approx$ $1 / \epsilon=10^{2}-10^{4}$. This is at least eleven times the maximum optical thickness of the line-forming region in our model, which is 8.9. So the source function thermalises in the photosphere, even when the line-forming region in the chromosphere is optically thick. We thus expect strong 3D effects on the source function.

We investigated this effect by comparing the full 3D computation with the 1D column-by-column computation. The top row of Fig. 8 shows joint probability density functions (JPDFs) of the 

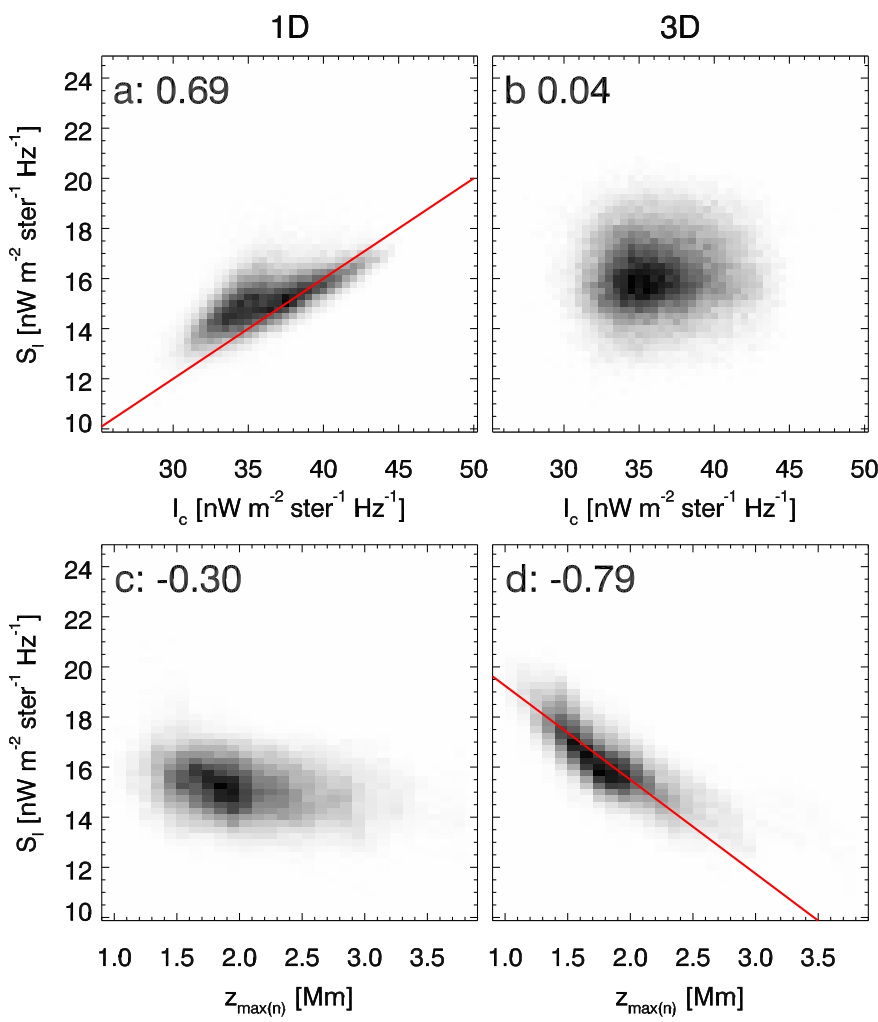

Fig. 8. JPDFs of the He I $1083 \mathrm{~nm}$ source function and the continuum intensity and the height where the line opacity is largest. The red line in panel a) is $S_{1}=0.4 I_{\mathrm{c}}$. The red line in panel d) is $S_{1}=23-3.75 z_{\max (n)}$

line source function $S_{1}$ and the underlying continuum intensity $I_{\mathrm{c}}$ computed from each $(x, y)$-pixel in our model, for both the 3D and 1D computation.

Panel a shows a strong correlation between $S_{1}$ and $I_{\mathrm{c}}$ in the $1 \mathrm{D}$ computation. The red line shows that roughly speaking, $S_{1}=0.4 I_{\text {c }}$. This is consistent with Avrett et al. (1994) who obtained the same relation for 1D semi-empirical models illuminated with a prescribed UV radiation field. In 3D this correlation is gone (panel b). The reason is that the source function at a given location in the chromosphere is no longer correlated to the continuum intensity from the photosphere directly below it, but by a spatial average of the continuum intensity emitted from an extended region encompassing multiple granules.

The bottom row of Fig. 8 shows the JPDFs for $S_{1}$ and the height where the lower-level population is maximum in the chromosphere $z_{\max (n)}$. In 1D there is just a rather weak correlation between the two quantities, which only shows up in the correlation coefficient. In $3 \mathrm{D}$ it is strong, and is clearly visible in the JPDF as well.

The behaviour of the He I $1083 \mathrm{~nm}$ source function is similar to the source function in the $\mathrm{H} \alpha$ line (Leenaarts et al. 2012). Both lines have source functions that thermalise in the photosphere, both lines are strongly scattering and both lines have negligible opacity in the upper photosphere and lower chromosphere.

\subsection{Relative importance of $3 D$ in the UV illumination and source function}

In order to assess the relative importance of $3 \mathrm{D}$ effects in the UV illumination and the source function we did a test calculation where we computed the emergent intensity using the opacity

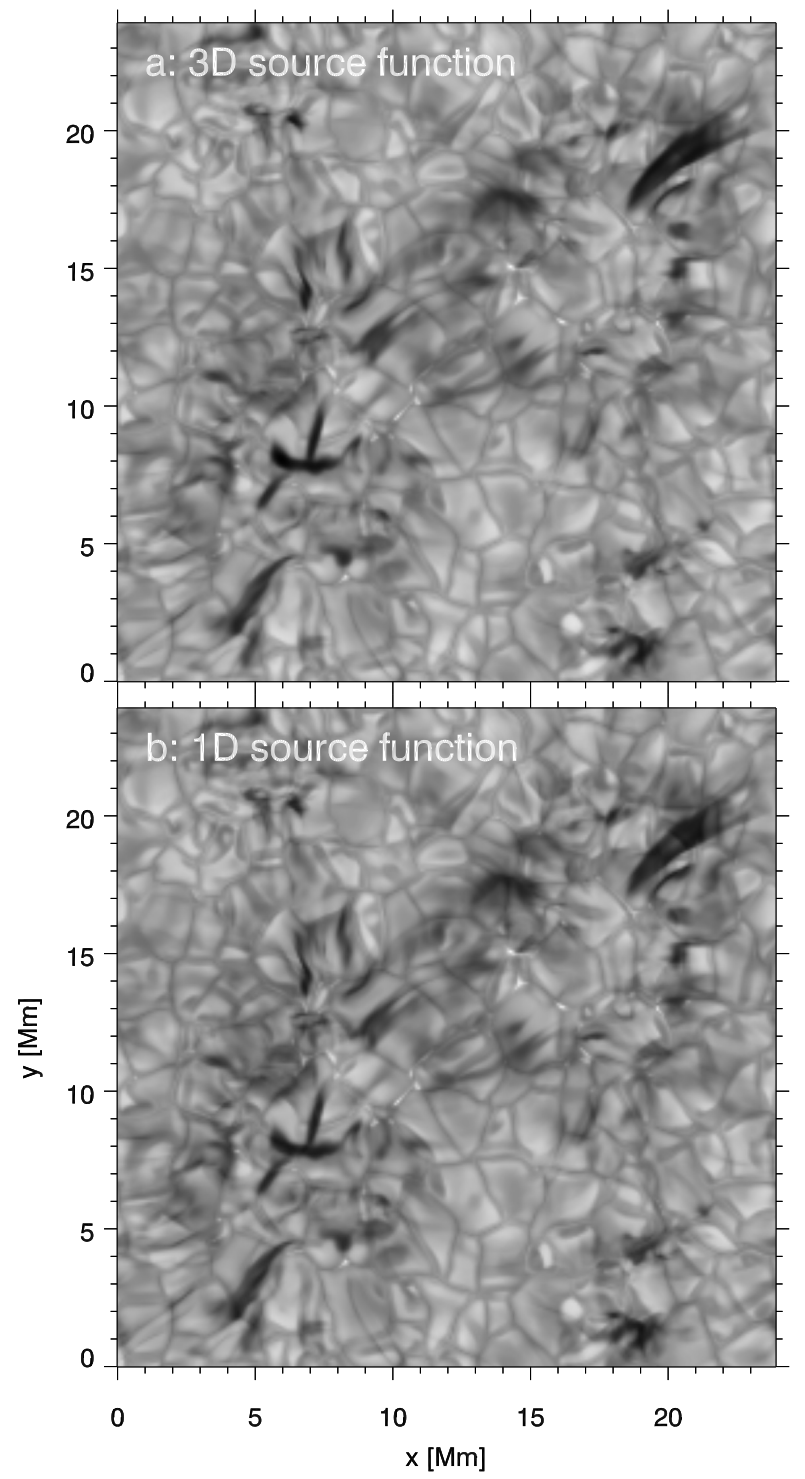

Fig. 9. Comparison of the synthetic He I $1083 \mathrm{~nm}$ line intensity computed with the correct 3D source function (upper panel) and an approximate 1D source function in the chromosphere (lower panel).

from the 3D computation, but used a line source function of the form

$S_{1}(x, y, z)=0.4 I_{\mathrm{c}}(x, y)$,

which was shown in Sect. 4.5 to be a decent approximation of the source function as obtained from 1D plane-parallel computations. The resulting line-centre intensity image, together with the $3 \mathrm{D}$ computation, is shown in Fig. 9. Both images are very similar in absorption structure, so we conclude that 3D effects in the UV illumination are the most important for the He I $1083 \mathrm{~nm}$ formation, while $3 \mathrm{D}$ effects on the line source function are minor.

\subsection{Variation in He I $1083 \mathrm{~nm}$ opacity and the chromospheric electron density}

In the previous subsections we explored the origin and effect of the ionising radiation. In this subsection we discuss the combined influence of the electron density and the radiation. Equation (1), which was derived by Livshits et al. (1976), predicts a 

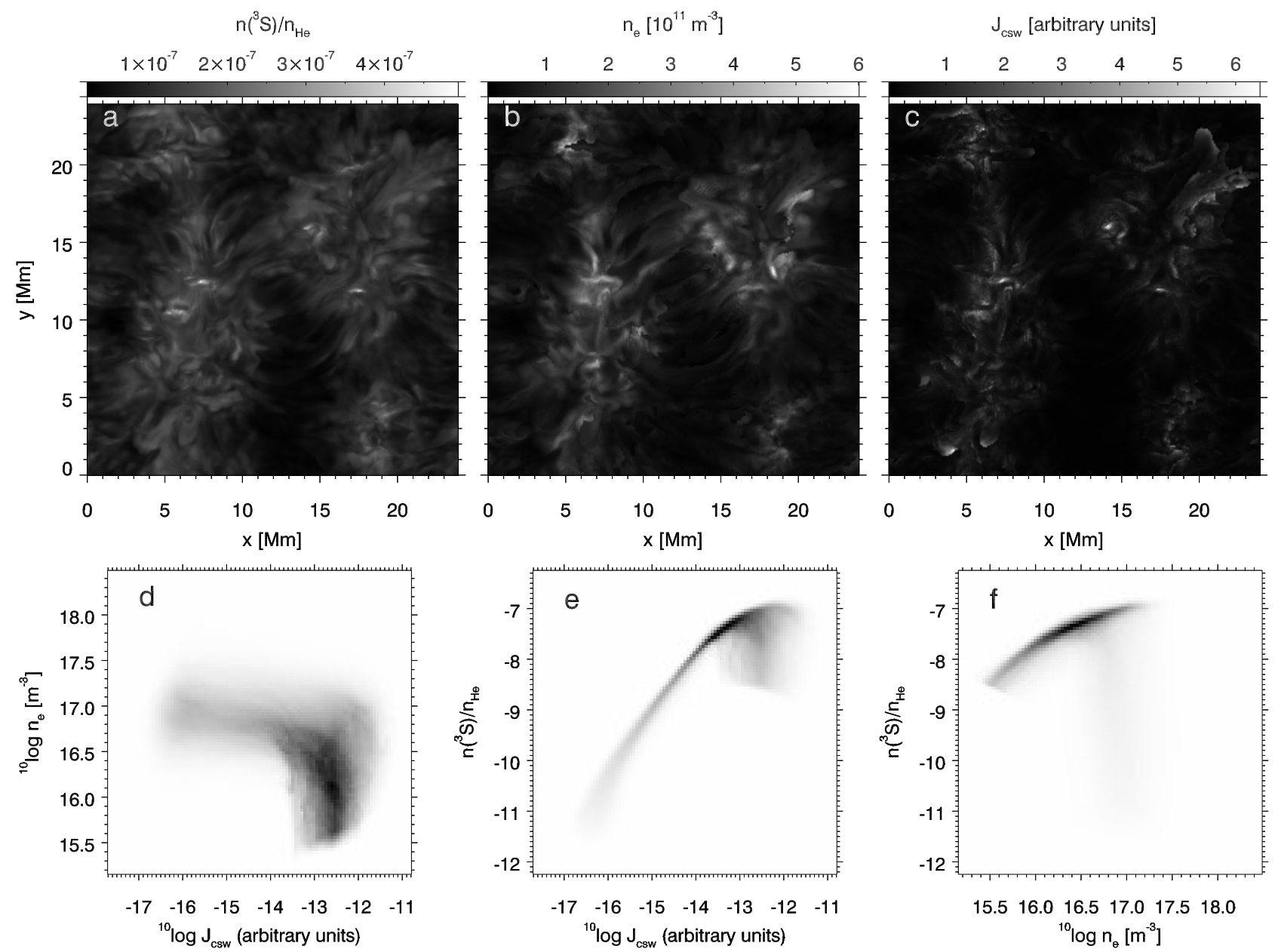

Fig. 10. Relation between He I $1083 \mathrm{~nm}$ opacity, electron density and the coronal irradiation. Panel a): relative population of the He I $1083 \mathrm{~nm}$ lower level, for each pixel at the height in the chromosphere where the relative population has a maximum. Panel b): electron density at the same location as in a). Panel c): $J_{\text {csw }}$ at the same location as in a) and b). Panel d): JPDF of $J_{\text {csw }}$ and the electron density in the chromosphere. Panel d): JPDF of $J_{\text {csw }}$ and the relative population of the He I $1083 \mathrm{~nm}$. Panel f): JPDF of the electron density and the relative population of the He I $1083 \mathrm{~nm}$.

correlation between the relative population $n\left(2 \mathrm{~s}^{3} \mathrm{~S}\right) / n_{\mathrm{He}}$ of the He I $1083 \mathrm{~nm}$, and those quantities. We illustrate this in Fig. 10. For each $(x, y)$ location in the model we looked up the location in the chromosphere where $n\left(2 \mathrm{~s}^{3} \mathrm{~S}\right) / n_{\mathrm{He}}$ has a maximum. For those locations we extracted $n\left(2 \mathrm{~s}^{3} \mathrm{~S}\right) / n_{\mathrm{He}}, n_{\mathrm{e}}$, and $J_{\mathrm{csw}}$ and display the resulting maps in panels a-c. The map of $n\left(2 \mathrm{~s}^{3} \mathrm{~S}\right) / n_{\mathrm{He}}$ is finely structured on small scales, but appears relatively smooth on larger scales. The electron density map shows a substantial correspondence with the $n\left(2 \mathrm{~s}^{3} \mathrm{~S}\right) / n_{\mathrm{He}}$ map. The map of $J_{\mathrm{csw}}$ in panel $\mathrm{c}$ is, in contrast, much more intermittent.

In panels $\mathrm{d}-\mathrm{f}$ we put the correlation on a more precise footing. Panel d shows the joint probability density function of $J_{\text {csw }}$ and $n_{\mathrm{e}}$. It shows a peculiar $\neg$-shape. Panel e shows the JPDF of $J_{\text {csw }}$ and $n\left(2 \mathrm{~s}^{3} \mathrm{~S}\right) / n_{\mathrm{He}}$. It consists of two distinct components, a tight positive correlation that roughly follows the prediction of Eq. 1 for fixed electron density and a beard-shaped cluster of points of $-13<\log _{10} J_{\text {csw }}<-12$. In panel $d$ it can be seen that the latter points correspond to a sudden large spread in values of the electron density, and thus a much weaker correlation between $J_{\text {csw }}$ and $n\left(2 \mathrm{~s}^{3} \mathrm{~S}\right) / n_{\mathrm{He}}$. A similar effect appears in the JPDF of $n_{\mathrm{e}}$ and $n\left(2 \mathrm{~s}^{3} \mathrm{~S}\right) / n_{\mathrm{He}}$ : there is a component with a tight correlation and for $16.5<\log _{10} n_{\mathrm{e}}<17$ there is a beard-like extension downward, caused by the large spread of values in $J_{\text {csw }}$ at those electron densities, as shown in panel $\mathrm{d}$.
We thus conclude that the approximate formula made by Livshits et al. (1976) is validated by our 3D non-LTE radiative transfer computations.

\section{Correlation with Si IV 1393 and 1402 and AIA 304, 171, 193 and 335 emission}

In Sect. 4.1 we showed that the source of the most intense He I ground state continuum radiation impinging on the chromosphere in our simulation comes from gas with a temperature of about $100 \mathrm{kK}$. This temperature is close to the coronalequilibrium peak formation temperature of the Si IV lines at $139.38 \mathrm{~nm}$ and $140.28 \mathrm{~nm}$, which is at $\log T=4.8 \mathrm{~K}$. These lines dominate the emission in the $140 \mathrm{~nm}$ slit-jaw imager of IRIS.

In Fig. 11 we compare a synthetic Si IV SJI image computed from our simulation snapshot and a map of the columnintegrated $L_{\mathrm{csw}}$, that is, $\int L_{\mathrm{csw}} \mathrm{d} z$. The brightest Si IV SJI emission corresponds to the brightest $L_{\mathrm{csw}}$, but the latter is more extended and has a diffuse component, owing to the large variety of lines that contribute to it.

The bottom panel of Fig. 11 shows a histogram of $L_{\mathrm{csw}}$ for all grid points in the simulation in black. In red it shows a histogram of $L_{\mathrm{csw}}$ for only those grid points that belong to the $0.1 \%$ grid 

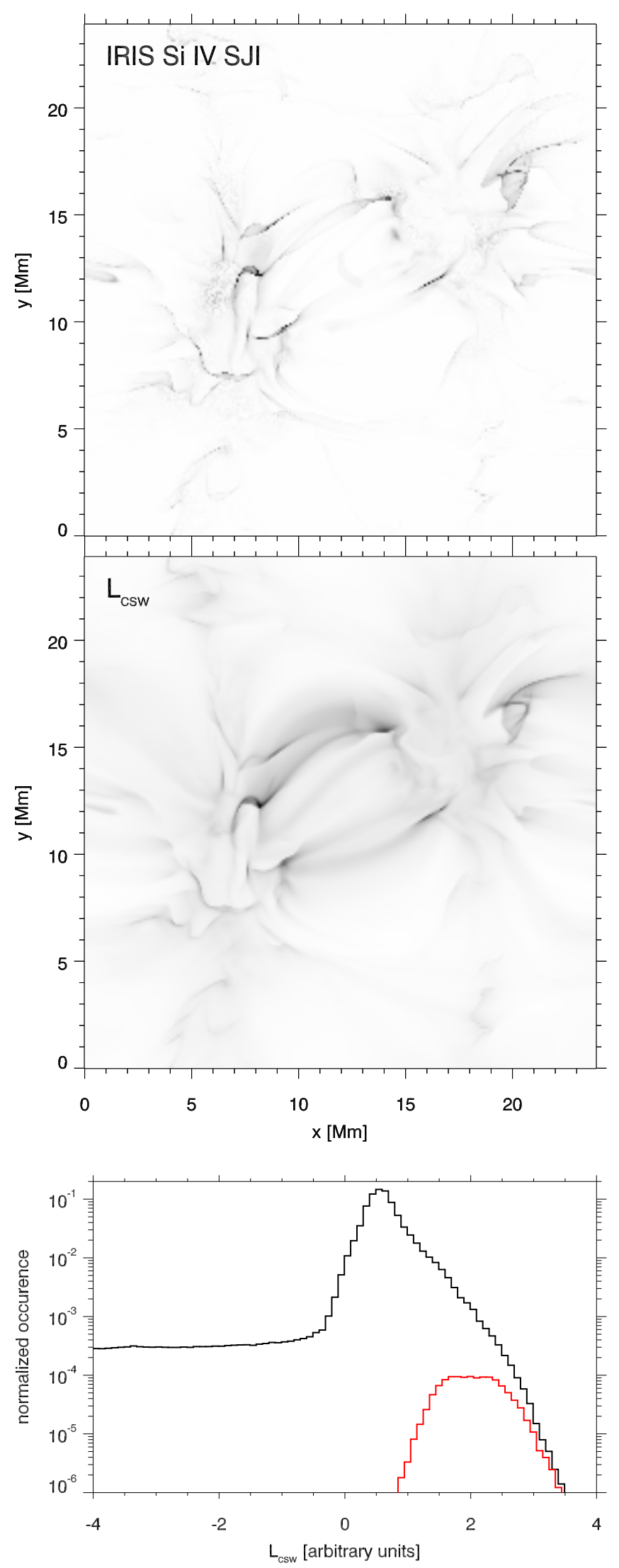

Fig. 11. Comparison of the synthetic IRIS $140 \mathrm{~nm}$ slit jaw emission (upper panel) and an image computed from $L_{\mathrm{csw}}$, the frequency integrated and cross-section weighted photon losses (middle panel). The bottom panel shows the histogram of $L_{\mathrm{csw}}$ for all grid points in the simulation (black) and only for the $0.1 \%$ of the grid points that have the highest Si IV emissivity (red). points that have the highest Si IV SJI emmisivity. This confirms quantitatively that locations with very high Si IV emission indeed correspond to locations with the highest $L_{\mathrm{csw}}$.

Based on the results shown in Fig. 11 and the rough correlation between $L_{\mathrm{csw}}$ and the lower-level population of He I 1083 nm (see Fig. 4), we thus expect a considerable correlation between Si IV emission and He I $1083 \mathrm{~nm}$ strength on scales of $\sim 1$ arcsec. We also show that the bulk of the ionizing photons are coming from hotter gas, emitted in a large volume in the overlying corona. We thus also expect a correlation between He I $1083 \mathrm{~nm}$ strength and coronal emission at larger scales. The latter correlation has of course been known for a long time (e.g., Kahler et al. 1983).

We therefore compared a single raster scan of the He I $1083 \mathrm{~nm}$ line taken with the TRIPPEL spectrograph at the SST with co-temporal and co-spatial artificial raster scans constructed from Si IV slit-jaw images and SDO/AIA images (see Sect. 3). Observationally we cannot directly determine atomic level populations, so we took the equivalent width $(\mathrm{EW})$ as a measure instead.

Panel a in Fig. 12 shows the raster scan in a continuum wavelength close to the He I $1083 \mathrm{~nm}$ line. There is a small sunspot with an irregular penumbra on the right side of the field-of-view, with granulation and some small pores filling the rest of the panel. Inspection of the IRIS $283.2 \mathrm{Mg}$ II $\mathrm{k}$ wing slit-jaw image (not shown) indicates that the observed area shows a considerable amount of magnetic bright points. Panel b shows an image of the He I $1083 \mathrm{~nm}$ core. In each pixel the intensity of the profile minimum is taken to compensate for Doppler shifts. The superpenumbra is clearly seen on the right side. The middle part of the image $\left(5<x<20^{\prime \prime}\right)$ is filled with fibrils with granulation visible in between. The left side of the image shows a more irregular absorption structure. The strong elongated absorption feature at the bottom of the image $\left(y<5^{\prime \prime}\right)$ is an active region filament. Panel c shows the EW of the He I $1083 \mathrm{~nm}$ line. The filament has the highest EW, while the areas showing granulation in Panel b have the lowest. Panel d shows the Si IV artificial raster scan. The sunspot is very dark, the filament is not visible, the rest of the image shows filamentary structures with medium brightness and more irregularly shaped patches of high brightness.

The simulation results presented in this paper are not representative for sunspots or filaments. The correlation that we expect on the basis of our simulation and radiative transfer modelling is most likely valid for quiet sun and network areas. We therefore focus on the most quiet area in the observation, roughly speaking where $x<20^{\prime \prime}$ and $y>5^{\prime \prime}$ (indicated by the blue box in Fig. 12).

Comparison of the brightest areas in panel d with the profileminimum intensity and EW of $\mathrm{He}$ I $1083 \mathrm{~nm}$ in panel $12 \mathrm{~b}$ and $12 \mathrm{c}$ shows many places where Si IV brightness coincides with a large $\mathrm{He} \mathrm{I} 1083 \mathrm{~nm} \mathrm{EW}$, for example at $(x, y)=$ $\left(14^{\prime \prime}, 19^{\prime \prime}\right),(x, y)=\left(7^{\prime \prime}, 12^{\prime \prime}\right)$ and $(x, y)=\left(3^{\prime \prime}, 23^{\prime \prime}\right)$. Careful inspection shows many more locations of good correlation. There are also locations where the correlation is not present, for example at $(x, y)=\left(10^{\prime \prime}, 23^{\prime \prime}\right)$ and $(x, y)=\left(11^{\prime \prime}, 28^{\prime \prime}\right)$.

Panel e shows the Si IV emission, smoothed to the same resolution as the AIA data to facilitate a more fair comparison to the emission in the various AIA channels shown in panels $\mathrm{f}-\mathrm{i}$, from the coolest 304 channel to the hot 335 channel.

Comparing the AIA 304 channel (panel d, which is most sensitive the He II $30.4 \mathrm{~nm}$ emission) to Si IV in panel c, we see that some structures can be recognized in both images, and there is a correlation on large scales. The Si IV image shows considerable more contrast and shows many more small patches of large 

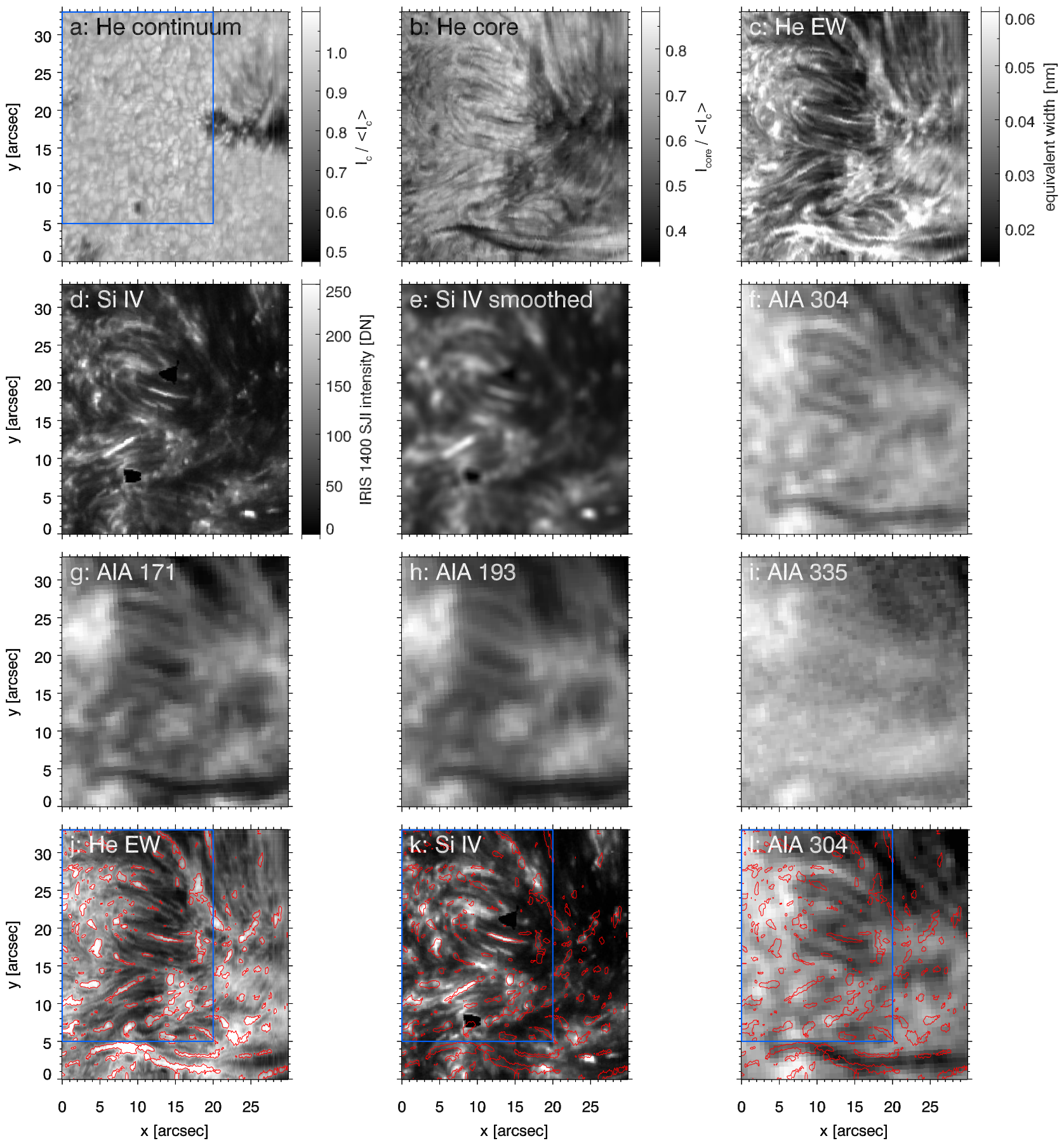

Fig. 12. Co-spatial and co-temporal observations of active region NOAA 12393 with the SST, IRIS and SDO/AIA. a) TRIPPEL raster scan image in the continuum at $1081.971 \mathrm{~nm}$. b) TRIPPEL raster scan image of the profile minimum of He I $1083 \mathrm{~nm}$. c) TRIPPEL raster scan image of the equivalent width of He I $1083 \mathrm{~nm}$. d) Artificial raster scan constructed from the IRIS $140 \mathrm{~nm}$ SJI images which are dominated by Si IV emission. e) same as d), but now smoothed to AIA resolution. f)-i) artificial raster scans for the AIA band given in the panels. j)-k): same as c), d), and f), but now with contours of local maxima in the EW overplotted in red. The blue box outlines the area discussed in the text.

brightness. The difference in appearance is not only caused by differences in spatial resolution between the IRS and SDO data. The large-scale patches of high EW in panel c correlate roughly with the high-brightness patches in 171 and 193, and to a lesser extent to the 304 and 335 images.

The bottom row (panels j-1) show the EW, Si IV, and 304 image again, but now overlaid with contours enclosing areas of local maxima in EW. These contours where created by thresholding a high-pass-filtered EW image, designed to pick up local maxima in EW, and serve to guide the eye. Inspection of panels $\mathrm{k}$ and 1 shows that the contours of local maxima in EW match the Si IV image much better than the 304 image.

Figure 13 compares the joint probability density function of the He I 1083 nm equivalent width and the Si IV brightness. For the observations we computed it from the area where $x<20^{\prime \prime}$ and $y<5^{\prime \prime}$ in Fig. 12. For the simulation we degraded the synthetic Si IV image to the spatial resolution of the observations. The observed correlation clearly shows the correlation between high equivalent width and Si IV brightness. For the simulations this correlation is not as strong. The correlation appears as the 

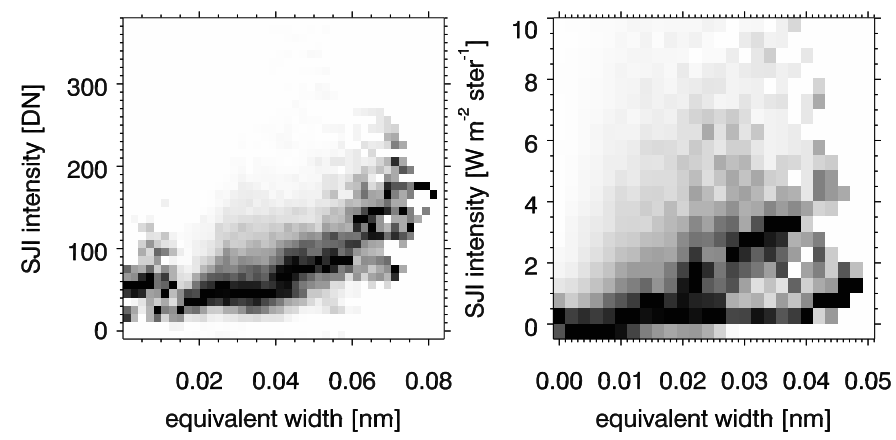

Fig. 13. Joint probability density functions of the equivalent width of He I $1083 \mathrm{~nm}$ and Si IV140 nm emission. Left-hand panel: observations; right-hand panel: simulations.

upper arm of the bifurcated distribution for equivalent widths larger than $0.22 \mathrm{~nm}$.

\section{Discussion and conclusions}

In this paper we investigated why the absorption features in images taken in the He I $1083 \mathrm{~nm}$ line in quiet Sun and network regions show intricate fine structuring to sub-arcsecond scale using a 3D radiation-MHD simulation of the solar atmosphere, combined with 3D non-LTE statistical equilibrium radiative transfer modelling.

We confirm the mechanism for populating the lower level of the line through photoionisation from the singlet ground state of He I to He II, followed by photorecombination into the He I triplet system as was originally put forward by Goldberg (1939). Direct collisional excitation at temperatures above $20 \mathrm{kK}$ is a negligible source of lower level population in our model. We also confirm the conclusion by Livshits et al. (1976) that the relative opacity of He I $1083 \mathrm{~nm}$ is electron density dependent: for a given mass density and coronal illumination the extinction coefficient is an increasing function of the electron density under typical chromospheric conditions.

Our simulations are not representative for strongly magnetically active areas and phenomena, such as emerging flux regions, flares and plage. These are typically associated with elevated chromospheric densities and temperatures and the presence of non-thermal electrons, where population of the triplet system can also occur through direct collisional excitation or collisional ionisation/recombination pathways (e.g., Ding et al. 2005). Our conclusions are therefore not necessarily valid under such circumstances.

We found that the sources of the ionising radiation can be roughly split in two components. The majority of the ionising photons are produced by gas in the $0.5-2 \mathrm{MK}$ temperature range. The source regions are extended as these are the temperatures of typical coronal gas, and their emissivity is relatively low. The second component consists of transition region gas between $80 \mathrm{kK}$ and $200 \mathrm{kK}$. It produces far fewer ionising photons than the high-temperature component. However, regions of high emissivity of the second component are concentrated in space and are located close to the upper chromosphere. This second, highly structured component is the main cause of variation in the UV flux impinging on the chromosphere. The actual radiation field $J_{\text {csw }}$ inside the chromosphere also depends on the shape of the transition region. Chromospheric material that is surrounded by a dense transition region has the highest $J_{\text {csw }}$ and consequently the highest He I ground state continuum photoionisation rate.

The He I $1083 \mathrm{~nm}$ line strength depends on the integral of the opacity along the line of sight. Therefore dense, verticallyoriented chromospheric structures that are surrounded by hot, dense, transition region and coronal material will typically produce the strongest line.

We tested our predicted correlation between the presence of high-mass-density $\approx 100 \mathrm{kK}$ gas and He I $1083 \mathrm{~nm}$ absorption by comparing emission in the Si IV $139.38 \mathrm{~nm}$ and $140.28 \mathrm{~nm}$ lines that form around $80 \mathrm{kK}$ with He I $1083 \mathrm{~nm}$ absorption and found that small-scale patches of high He I $1083 \mathrm{~nm}$ EW indeed often correlate with high Si IV emission. The correlation is notably absent around the active region filament in our observations, which might indicate a thin and/or low-mass-density transition region around the filament. The correlation between small-scale structure in the EW and AIA 304 is significantly worse. The largescale variation in EW correlates well with the emission in coronal lines as sampled by the AIA 171 and 193 as well as the AIA 304 emission.

Our work is an improvement over earlier modelling efforts (e.g., Zirin 1975; Pozhalova 1988; Avrett et al. 1994; Centeno et al. 2008). We used a 3D radiation-MHD simulation of the solar atmosphere, as opposed to the 1D models used before. We self-consistently include the emission of ionising radiation from the corona: it is produced by the corona present in the radiation-MHD simulation. Earlier work used a prescribed coronal radiation field.

Despite these improvements there are still a number of limitations to our approach: the simulation that we use produces too little chromospheric dynamics and produces to little chromospheric emission compared to observations (see for example Carlsson et al. 2016; Leenaarts et al. 2013). Increasing the numerical resolution and adding heating effects from ion-neutral interactions (Martínez-Sykora et al. 2012; Khomenko \& Collados 2012) might alleviate some of the discrepancy in future simulations. We also base our current analysis on a single snapshot, with a single magnetic field configuration. A future improvement would be to repeat the analysis for a time series of snapshots and for simulation runs with different magnetic field geometries.

The biggest discrepancy in our current analysis is that we do not include the effect of non-equilibrium ionisation in our 3D non-LTE radiative transfer calculation for helium, nor do we include non-equilibrium ionisation effects on the production of ionising photons.

Non-equilibrium ionisation in the transition region and corona typically has the effect that ions in a particular ionisation stage are present over a larger temperature range than what one would expect from coronal equilibrium calculations (Olluri et al. 2013b). The effect on the resulting emissivities and intensities are rather mild for the lines of SiIV, CIV, and O IV that were investigated by Olluri et al. (2015). No non-equilibrium intensity calculations for the lines dominating the He I photoionisation mentioned in at the end of Sect. 4.1 have been done, but the results for the non-equilibrium ionisation balance of oxygen from Olluri et al. (2013a) indicate that O III lines should experience similar mild effects as the O IV lines. Non-equilibrium effects also play a role for the He II $30.4 \mathrm{~nm}$ line (e.g. Golding et al. 2014). However, we showed that the line produces roughly the right amount of photons, so that neglecting this effect has little effect on the formation of the He I $1083 \mathrm{~nm}$ line.

Comparisons of one-dimensional calculations assuming SE and NE for helium using the RADYN code (e.g., 
Carlsson \& Stein 1992) indicate that large differences in the He I $1083 \mathrm{~nm}$ profiles can occur (Golding et al. 2014). While we expect that inclusion of non-equilibrium effects will have large quantitative effects on He I $1083 \mathrm{~nm}$ formation, we do not think it will have a qualitative effect on the spatial structure: also in the non-equilibrium case the photoionisation-recombination mechanism is the dominant mechanism populating the triplet states (Golding et al. 2014). The correlation between He I $1083 \mathrm{~nm}$ absorption and strong emission in the transition region and high chromospheric electron density will thus remain valid, even though the contribution of direct collisional excitation due to the presence of neutral helium at higher temperatures might play a bigger role. Because we expect quantitative differences when non-equilibrium radiative transfer for helium is taken into account we have refrained from detailed analysis of the synthetic He I $1083 \mathrm{~nm}$ profiles in this work.

Acknowledgements. This research was supported by the Research Council of Norway through the grant "Solar Atmospheric Modelling" and through grants of computing time from the Programme for Supercomputing. The research leading to these results has received funding from the European Research Council under the European Union's Seventh Framework Programme (FP7/2007-2013)/ERC grant agreement No. 291058. Some computations were performed on resources provided by the Swedish National Infrastructure for Computing (SNIC) at the National Supercomputer Centre at Linköping University. The Swedish 1-m Solar Telescope is operated on the island of La Palma by the Institute for Solar Physics of the Royal Swedish Academy of Sciences in the Spanish Observatorio del Roque de los Muchachos of the Instituto de Astrofísica de Canarias. IRIS is a NASA small explorer mission developed and operated by LMSAL with mission operations executed at NASA Ames Research center and major contributions to downlink communications funded by ESA and the Norwegian Space Centre.

\section{References}

Andretta, V., \& Jones, H. P. 1997, ApJ, 489, 375

Auer, L. 2003, in Stellar Atmosphere Modeling, ASP Conf. Ser., 288, 3

Avrett, E. H., Fontenla, J. M., \& Loeser, R. 1994, Formation of the solar 10830

A line Infrared Solar Physics, eds. D. M. Rabin, J. T. Jefferies, \& C. Lindsey (Dordrecht: Kluwer) IAU Symp. 154, 35

Bradshaw, S. J., \& Raymond, J. 2013, Space Sci. Rev., 178, 271

Carlson, B. G. 1963, Meth. Comput. Phys., 1

Carlsson, M., \& Leenaarts, J. 2012, A\&A, 539, A39

Carlsson, M., \& Stein, R. F. 1992, ApJ, 397, L59

Carlsson, M., \& Stein, R. F. 2002, ApJ, 572, 626

Carlsson, M., Hansteen, V. H., Gudiksen, B. V., Leenaarts, J., \& De Pontieu, B. 2016, A\&A, 585, A4

Centeno, R., Trujillo Bueno, J., Uitenbroek, H., \& Collados, M. 2008, ApJ, 677, 742

Cirtain, J. W., Golub, L., Winebarger, A. R., et al. 2013, Nature, 493, 501

de la Cruz Rodríguez, J., Löfdahl, M. G., Sütterlin, P., Hillberg, T., \& Rouppe van der Voort, L. 2015, A\&A, 573, A40
De Pontieu, B., Title, A. M., Lemen, J. R., et al. 2014, Sol. Phys., 289, 2733

Dere, K. P., Landi, E., Mason, H. E., Monsignori Fossi, B. C., \& Young, P. R. 1997, A\&AS, 125, 149

Ding, M. D., Li, H., \& Fang, C. 2005, A\&A, 432, 699

Fontenla, J. M., Avrett, E., Thuillier, G., \& Harder, J. 2006, ApJ, 639, 441

Goldberg, L. 1939, ApJ, 89, 673

Golding, T. P., Carlsson, M., \& Leenaarts, J. 2014, ApJ, 784, 30

Golding, T. P., Leenaarts, J., \& Carlsson, M. 2016, ApJ, 817, 125

Gudiksen, B. V., Carlsson, M., Hansteen, V. H., et al. 2011, A\&A, 531, A154

Gustafsson, B. 1973, Uppsala Astr. Obs. Ann., 5, 6

Hansteen, V. 1993, ApJ, 402, 741

Ibgui, L., Hubený, I., Lanz, T., \& Stehlé, C. 2013, A\&A, 549, A126

Ji, H., Cao, W., \& Goode, P. R. 2012, ApJ, 750, L25

Jordan, C. 1975, MNRAS, 170, 429

Joselyn, J. A., Munro, R. H., \& Holzer, T. E. 1979, ApJS, 40, 793

Kahler, S. W., Davis, J. M., \& Harvey, J. W. 1983, Sol. Phys., 87, 47

Khomenko, E., \& Collados, M. 2012, ApJ, 747, 87

Kiselman, D., Pereira, T. M. D., Gustafsson, B., et al. 2011, A\&A, 535, A14

Landi, E., Young, P. R., Dere, K. P., Del Zanna, G., \& Mason, H. E. 2013, ApJ, 763,86

Leenaarts, J., \& Carlsson, M. 2009, in The Second Hinode Science Meeting: Beyond Discovery-Toward Understanding, eds. B. Lites, M. Cheung, T. Magara, J. Mariska, \& K. Reeves, ASP Conf. Ser., 415, 87

Leenaarts, J., Carlsson, M., Hansteen, V., \& Rutten, R. J. 2007, A\&A, 473, 625 Leenaarts, J., Carlsson, M., \& Rouppe van der Voort, L. 2012, ApJ, 749, 136 Leenaarts, J., Pereira, T. M. D., Carlsson, M., Uitenbroek, H., \& De Pontieu, B. 2013, ApJ, 772, 90

Lemen, J. R., Title, A. M., Akin, D. J., et al. 2012, Sol. Phys., 275, 17

Livshits, M. A., Akimov, L. A., Belkina, I. L., \& Diatel, N. P. 1976, Sol. Phys., 49, 315

MacPherson, K. P., \& Jordan, C. 1999, MNRAS, 308, 510

Martínez-Sykora, J., De Pontieu, B., \& Hansteen, V. 2012, ApJ, 753, 161

Milkey, R. W., Heasley, J. N., \& Beebe, H. A. 1973, ApJ, 186, 1043

Olluri, K., Gudiksen, B. V., \& Hansteen, V. H. 2013a, ApJ, 767, 43

Olluri, K., Gudiksen, B. V., \& Hansteen, V. H. 2013b, AJ, 145, 72

Olluri, K., Gudiksen, B. V., Hansteen, V. H., \& De Pontieu, B. 2015, ApJ, 802, 5

Pereira, T. M. D., Kiselman, D., \& Asplund, M. 2009, A\&A, 507, 417

Pesnell, W. D., Thompson, B. J., \& Chamberlin, P. C. 2012, Sol. Phys., 275, 3 Pietarila, A., \& Judge, P. G. 2004, ApJ, 606, 1239

Pozhalova, Z. A. 1988, Sov. Astron., 32, 542

Rybicki, G. B., \& Hummer, D. G. 1991, A\&A, 245, 171

Rybicki, G. B., \& Hummer, D. G. 1992, A\&A, 262, 209

Sanz-Forcada, J., \& Dupree, A. K. 2008, A\&A, 488, 715

Schad, T. A., Penn, M. J., \& Lin, H. 2013, ApJ, 768, 111

Schad, T. A., Penn, M. J., Lin, H., \& Tritschler, A. 2015, Sol. Phys., 290, 1607

Scharmer, G. B., Bjelksjo, K., Korhonen, T. K., Lindberg, B., \& Petterson, B.

2003, in Innovative Telescopes and Instrumentation for Solar Astrophysics,

eds. S. L. Keil, \& S. V. Avakyan, SPIE Conf. Ser., 4853, 341

Smith, G. R. 2003, MNRAS, 341, 143

Smith, G. R., \& Jordan, C. 2002, MNRAS, 337, 666

Tobiska, W. K. 1991, J. Atmospheric Terrestrial Phys., 53, 1005

Tobiska, W. K. 2004, Adv. Space Res., 34, 1736

Woods, T. N., Eparvier, F. G., Hock, R., et al. 2012, Sol. Phys., 275, 115

Zarro, D. M., \& Zirin, H. 1986, ApJ, 304, 365

Zirin, H. 1975, ApJ, 199, L63 